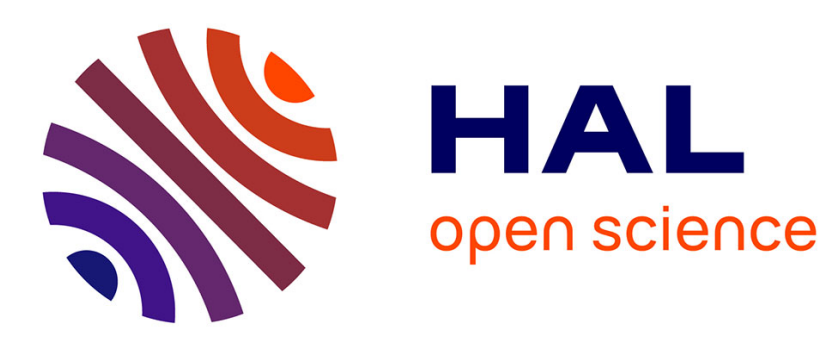

\title{
L'intermédiation financière dans l'analyse macroéconomique: Le défi de la crise
}

Eleni Iliopulos, Thepthida Sopraseuth

\section{To cite this version:}

Eleni Iliopulos, Thepthida Sopraseuth. L'intermédiation financière dans l'analyse macroéconomique:

Le défi de la crise. 2011. halshs-00613188

\section{HAL Id: halshs-00613188 \\ https://shs.hal.science/halshs-00613188}

Submitted on 3 Aug 2011

HAL is a multi-disciplinary open access archive for the deposit and dissemination of scientific research documents, whether they are published or not. The documents may come from teaching and research institutions in France or abroad, or from public or private research centers.
L'archive ouverte pluridisciplinaire HAL, est destinée au dépôt et à la diffusion de documents scientifiques de niveau recherche, publiés ou non, émanant des établissements d'enseignement et de recherche français ou étrangers, des laboratoires publics ou privés. 


\section{Documents de Travail du Centre d'Economie de la Sorbonne}

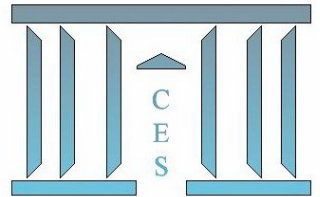

L'intermédiation financière dans l'analyse macroéconomique : le défi de la crise

Eleni ILIOPULOS, Thepthida SOPRASEUTH

2011.46

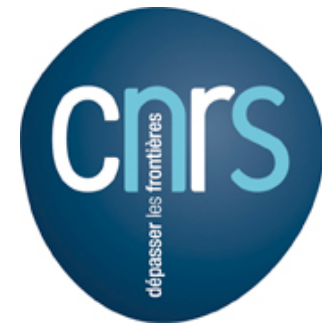




\title{
L’intermédiation financière dans l’analyse macroéconomique : Le défi de la crise
}

\author{
Eleni Iliopulos \\ PSE, Université de Paris I et CEPREMAP
}

Thepthida Sopraseuth

GAINS-TEPP, Université du Maine et CEPREMAP

Version soumise : Novembre 2010

Version révisée : Juillet $2011^{1}$

\begin{abstract}
In this paper, we review the macroeconomic literature on financial frictions and banking in a dynamic general equilibrium framework. Our work focuses first on the pioneer articles that have analyzed the amplification effects associated to the financial accelerator. We then shift our attention towards the recent literature that flourished in the aftermath of the financial crisis. Indeed, the crisis has challenged several assumptions and modeling tools that were commonly used in the DSGE literature. We thus review the main recent contributions that have tried to overcome the limits of old models.
\end{abstract}

JEL codes: E3, E4, E5, G01, G21.

Keywords: financial frictions, banking, monetary policy, business cycle.

\footnotetext{
${ }^{1}$ Nous tenons à remercier les rapporteurs, l'éditeur et L. Patureau pour leur lecture attentive. Nous avons également bénéficié de discussions avec J. Couppey-Soubeyran, P. Karadi, N. Kiyotaki, A. Queralto et M. Woodford.
} 


\section{Introduction}

La récente crise financière a brusquement interrompu une longue période de stabilité économique. Les économistes ont appelé la chute de la volatilité macroéconomique constatée dans les pays occidentaux depuis la fin des années 90 la "grande modération » (Bernanke, 2004 ; Stock et Watson, 2002). Cette dernière a été associée à des politiques économiques visant à stabiliser l'inflation. Un contexte économique caractérisé par des politiques crédibles donc prévisibles associées à la "grande modération» fut propice au développement d'ambitieux outils quantitatifs tels que les MEGIS (Modèle d'Equilibre Général Intertemporels et Stochastiques). La crise a remis en question la capacité de ces outils à rendre compte et à prévoir les fluctuations.

Dans cet article, nous présentons la modélisation du secteur financier dans la littérature des MEGIS. Ces maquettes de l'économie visent à quantifier l'impact sur les variables d'intérêt (inflation, prix des actifs, PIB, taux d'intérêt par exemple) de l'ensemble des interactions des agents économiques présents dans le modèle. Cet accent sur l'équilibre général implique une modélisation lourde intégrant non seulement le comportement de tous les agents, mais également l'impact de l'ensemble de ces comportements sur le prix de chaque marché modélisé dans la maquette. Un économiste raisonnant en équilibre partiel ne s'intéresserait qu'aux comportements individuels sans rechercher à quantifier l'impact des interactions individuelles sur l'économie. L'approche en équilibre général s'impose en particulier dans l'analyse de la crise récente au cours de laquelle les choix des agents économiques ont affecté les prix de marché, lesquels viennent, dans un effet retour, modifier les actions des agents. On peut évoquer par exemple, la crise de liquidité sur le marché interbancaire intervenue dès l'été 2007 aux Etats-Unis, qui a engendré un relèvement du taux d'intérêt interbancaire, cette dernière aggravant à son tour les difficultés de financement des acteurs du système financier.

Le développement des MEGIS doit concilier l'intégration de raffinements de modélisation (tels que ceux présentés dans cet article) et la nécessité de proposer une maquette calculable. La confrontation des prédictions quantitatives des MEGIS aux évolutions macroéconomiques observées dans les données éclairera l'économiste sur la pertinence des modèles proposés.

A la lumière de la crise, l'accent sur les MEGIS peut sembler peu judicieux. En effet, selon Miller et Stiglitz (2010), le paradigme moderne manque d'une bonne modélisation des agents hétérogènes, de l'asymétrie d'information, des externalités, de jeux de coordination et du défaut de paiement. Wickens (2009), Buiter (2009) et Caballero (2010) critiquent vivement l'approche de la macroéconomie moderne qui néglige les agents hétérogènes, les marchés incomplets ou imparfaits, le risque et les asymétries d'information. De Grauwe (2009) et Akerlof et Shiller (2009) dénoncent l'hypothèse d'anticipations rationnelles retenue dans les MEGIS. Nous retenons de ces critiques une invitation à dépasser la modélisation MEGIS existante. Certaines contributions présentées dans cet article intègrent des agents hétérogènes (Kiyotaki et Moore, 1997 ; Curdia et Woodford, 2010), visent à évaluer les conséquences des asymétries d'information (Bernanke, Gilchrist et Gertler, 1999; la section sur le capital bancaire) ou tiennent mieux compte du risque dans la décision des agents économiques (section sur le canal de la prise de risque).

Caballero (2010) définit l'évolution de la littérature comme une tension entre le « cœur» et la « périphérie ». La périphérie se nourrit de champs de recherche connexes tels que les théories financières ou la modélisation des décisions en univers incertain. Le cœur vise à développer des analyses quantitatives dans un cadre d'équilibre général dynamique, ce qui incite 
l'économiste à retenir des hypothèses simplificatrices facilitant la résolution de ces modèles complexes. Nous présentons dans cet article les contributions récentes qui tentent de se nourrir des enseignements de la périphérie pour améliorer les prévisions du cœur.

La crise récente a invité les économistes à réévaluer la modélisation du secteur financier dans les MEGIS. Il s'agit de proposer une véritable modélisation de l'intermédiation financière et de son impact macroéconomique afin de mesurer l'efficacité de la gestion de la crise choisie par les banques centrales. Ces dernières ont mené des politiques monétaires volontaristes avec une baisse marquée des taux directeurs. Toutefois, cette politique monétaire conventionnelle s'est heurtée à un taux plancher (zero interest rate bound). Les taux directeurs étant très bas, les banques centrales ont été dans l'impossibilité de les baisser davantage. Des politiques monétaires dites « non conventionnelles » ont été mises en œuvre afin de juguler la crise. Ces dispositifs, mis en place en période d'inefficacité de la politique conventionnelle de fixation des taux directeurs, comprennent une augmentation massive de la quantité de monnaie en circulation dans l'économie (" quantitative easing ») et un assouplissement des conditions de financement aux agents financiers et non financiers, la banque centrale se substituant aux marchés et aux banques commerciales pour financer l'économie («credit easing ») (pour une description de ces politiques non conventionnelles, voir CAE 2008 ; Bernanke, 2009 ; Banque de France, 2009 ; IMF, 2009)

La section 2 est consacrée aux 2 articles fondateurs d'accélérateur financier. La crise a suscité un foisonnement de travaux de recherche. La section 3 présente une sélection de contributions qui nous semblent représentatives des pistes de recherche prometteuses car elles tentent de relever les défis suscités par la crise (section 3.1.) et s'intègrent dans des canaux de transmission de la politique monétaire identifiés dans la littérature tels que le canal du capital bancaire (section 3.2.2.) et le canal de la prise de risque (section 3.2.3.). De plus, nous avons retenu des articles qui fournissent des évaluations quantitatives car, comme nous le soulignons en section 3.1., la crise présente un défi quantitatif pour la modélisation macroéconomique. La section 4 présente les recommandations en termes de politique économique.

\section{L'accélérateur financier}

Les premiers travaux sur l'accélérateur financier (Kiyotaki et Moore, 1997, ci-après KM ; Bernanke, Gertler et Gilchrist, 1999, ci-après BGG) visent à mieux comprendre les effets réels de la politique monétaire. L'accélérateur financier fait référence au mécanisme par lequel les contraintes de financement affectent les fluctuations macroéconomiques. Dans ce cas, le théorème de Modigliani-Miller, selon lequel, dans un monde parfait (marchés complets, absence de taxes, information parfaite), la valeur d'une firme ne dépend pas de la structure de son financement (capitaux propres ou financement externe), n'est pas vérifié. Les évolutions des conditions de financement, en affectant le montant de la dette et donc la structure de financement de la firme, ont des effets réels. Nous soulignerons dans cette section les apports et les limites de ces modèles que les développements récents visent à dépasser. ${ }^{2}$

\footnotetext{
${ }^{2}$ Des présentations plus détaillées de ces deux modèles fondateurs sont disponibles sur la pageweb des auteurs. Le lecteur y trouvera les équations des modèles, l'étalonnage et les programmes DYNARE à l'origine des graphiques de cette section.
} 


\section{1. Un effet d'amplification via le prix des actifs : Kiyotaki et Moore (1997)}

Le travail de KM constitue l'une des premières analyses d'équilibre général qui s'intéressent aux effets d'amplification liés aux frictions financières. On entend par frictions financières l'ensemble des caractéristiques dans le modèle qui font obstacle au flux financiers entre les agents qui disposent de fonds et ceux en besoin de financement.

De plus, ce cadre développe des éléments qui nous permettent d'expliquer plusieurs faits stylisés afférents à la période antérieure à la crise de 2008. Cette période a été caractérisée par un boom de l'immobilier associé, dans plusieurs pays avancés, à une hausse de la consommation $^{3}$. Or, la corrélation positive entre la consommation privée et la richesse immobilière ${ }^{4}$ semble liée à la possibilité d'accéder au crédit immobilier ainsi qu'aux caractéristiques spécifiques du marché du crédit dans chaque pays (voir Calza et al., 2009). Le cadre de KM met l'accent sur le rôle des contraintes de crédit qui prennent la forme plus spécifiquement d'une contrainte de nantissement des prêts (collateral). La dynamique repose sur le mécanisme suivant: le niveau d'endettement des agents est borné par la valeur de leurs biens immobiliers (collateral). Une augmentation de la valeur et/ou de la quantité de ces derniers permet aux agents de s'endetter et de consommer davantage. Le modèle reproduit donc une corrélation positive entre consommation et actifs immobiliers, comme cela a été observé dans la période précédant la crise de 2008.

\subsubsection{Le modèle}

Dans le modèle de KM, l'économie est composée de deux types de ménages. Les premiers, appelés les banquiers, disposent à l'équilibre d'une épargne qu'ils prêteront au $2^{\text {nd }}$ type de ménage, en besoin de financement, appelés les entrepreneurs. Ces derniers produisent des biens (non durables) grâce au travail fourni par l'ensemble des ménages et au capital dont le stock s'accroît grâce à l'investissement. La production des entrepreneurs de biens non durables constitue le PIB de l'économie.

L'hypothèse centrale du modèle repose sur la contrainte d'endettement subie par les entrepreneurs pour financer leurs projets d'investissement et leur consommation. Le montant de la dette augmentée des paiements d'intérêt ne peut excéder une limite de crédit, définie comme une fraction exogène de la valeur du collateral.

On suppose donc que, à chaque période, la contrainte de collateral a la forme suivante ${ }^{5}$ :

$$
R_{t} B_{\tau} \simeq m E_{t}\left\{Q_{t+1} h_{\tau}^{*}\right\}
$$

où $R$ désigne le facteur d'intérêt, $B$ la dette nominale envers les banquiers, $h^{e}$ le collateral, $\mathrm{Q}$ le prix de marché du collateral et $m$ est un paramètre exogène ${ }^{6}$ compris entre 0 et 1 représentant la fraction de la valeur du collateral qui peut être empruntée. La valeur du

\footnotetext{
${ }^{3}$ Pendant la première partie de la dernière décennie, la propension de long terme à consommer la richesse immobilière été environ 0,01 en France, 0,07 en Angleterre et 0,15 aux Etats Unis (IMF, 2008).

${ }^{4}$ En France, la corrélation entre les prix de l'immobilier et la consommation courante été d'environ 0,1 (écart à la tendance en pourcentage), environ 0,45 aux Etats Unis et 0,6 en Angleterre (IMF, 2008).

5 En divisant par le prix du bien de consommation, on peut réécrire la contrainte en termes réels: $R_{t} b_{i} \leq m E_{z}\left\{\pi_{t+1} q_{i+1} h_{i}^{2}\right\}$, où les variables minuscules sont exprimées en termes réels et $\pi$ le facteur d'inflation. Cette expression fait apparaître le rôle de l'inflation dans la contrainte d'endettement.

${ }^{6}$ En France, la valeur de ce paramètre été estimé à 75\% pendant la période : 2003-2006 (voir IMF, 2008). Ce même paramètre été estimé à $75 \%$ pour l'Angleterre et $80 \%$ aux Etats Unis.
} 
paramètre $m$ est choisie de façon à résumer la contrainte d'endettement pertinente dans l'économie.

Cette hypothèse appelle plusieurs commentaires.

1) sur le fonctionnement du mécanisme d'accélérateur financier : La valeur du collateral est celle obtenue au prix de marché Q. Une hausse du prix de marché, en augmentant la valeur du collateral, permet aux entrepreneurs de s'endetter davantage. Ce surcroît de financement est utilisé par les entrepreneurs pour investir et consommer.

2) sur la nature du collateral intervenant dans la contrainte d'endettement: dans le modèle de $\mathrm{KM}$, le collateral désigne le stock de biens durables détenus par le ménage-entrepreneur, que la banque peut saisir en cas de faillite. Dans KM, ces biens durables sont la terre, dans Iacoviello (2005), ces biens durables sont les biens immobiliers. Cette hypothèse semble cohérente avec les données. La richesse immobilière, définie comme la valeur de marché du stock de biens immobiliers, représente aujourd'hui plus de la moitié de la richesse des ménages des pays développés ${ }^{7}$; aux Etats Unis, elle est également plus importante que le PIB. ${ }^{8}$ En outre, la richesse immobilière et la consommation semblent corrélées. ${ }^{9}$ Les études empiriques montrent également que les phases hautes et basses de l'investissement en actifs immobiliers précèdent les phases hautes et basses du cycle économique (données américaines sur la période 1952-2008 ; voir Iacoviello, 2010). En ce sens, comme suggéré par Ed Leamer, « l'immobilier est le cycle lui-même».

3) sur le fondement d'une telle contrainte: pour KM, la contrainte est justifiée par l'impossibilité pour la banque d'obliger les emprunteurs à rembourser leurs dettes (limited enforcement). Cela se traduit par un accès au crédit limité par la valeur des biens immobiliers. Cette contrainte de collateral peut être simplement interprétée comme une contrainte de solvabilité standard : lors de la demande de crédit, la banque refuse de financer la totalité de l'achat immobilier pour lequel le prêt est sollicité. La contrainte d'endettement est cohérente avec les critères d'endettement sur le marché du crédit et assure que le paiement des intérêts et le remboursement du principal n'excèdent pas une fraction (supposée ici exogène et égale à m) de la valeur des biens immobiliers. Notons également que, la contrainte d'endettement implique un levier constant, égal à $m$, quelle que soit la conjoncture économique. Or, les fluctuations du levier peuvent également amplifier les chocs agrégés. BGG explorent ce point.

4) sur l'implication en termes de résolution du modèle

KM retiennent une hypothèse supplémentaire afin de simplifier la résolution du modèle. En effet, a priori, cette limite de crédit n'est pas nécessairement saturée à toutes les périodes, ce qui rend la résolution du modèle complexe car non -linéaire (modèle avec contrainte occasionnellement saturée). Afin de s'assurer que la contrainte de crédit est saturée à toutes les périodes (l'entrepreneur a intérêt à s'endetter à chaque période à hauteur de sa limite de crédit), KM font l'hypothèse d'une hétérogénéité des taux d'escompte des agents. Les ménages sont plus patients que les entrepreneurs. Etant donné leur impatience relative, les préférences des entrepreneurs favorisent la consommation courante : leur utilité marginale de la consommation courante est supérieure à celle de l'épargne. L'hétérogénéité des taux

\footnotetext{
${ }^{7}$ Voir Iacoviello (2010).

${ }^{8}$ Sur la période 1952-2008, ce ratio aux Etats-Unis s’élevait à 1,5 (voir Iacoviello, 2010).

9 Aux Etats-Unis, la corrélation contemporaine entre consommation et richesse immobilière s'est élevée à 0,47 sur la période 1952-2008
} 
d'impatience assure que, à l'équilibre, les entrepreneurs sont toujours endettés envers les autres ménages, ceux-ci agissant en tant que banquiers.

L'hypothèse d'hétérogénéité des taux d'escompte est courante dans la littérature (Lawrance, 1991 ; Krussel et Smith, 1998 ; Calstrom et Fuerst 1997, 1998 ; Solomon 2010 ; Ghironi, Iscan et Rebucci en économie ouverte, 2008). Des études confirment la pertinence empirique de cette hypothèse (Cagetti, 2003), en particulier lorsque l'hypothèse d'hétérogénéité des taux d'escompte peut s'interpréter comme la forme réduite d'un cycle de vie (les agents impatients étant en début de cycle de vie ; Browning et Tobacman, 2007 ; Carroll, 2000)

\section{5) sur l'absence de faillite des emprunteurs}

La contrainte d'endettement qui plafonne la dette à une proportion de la valeur du nantissement du prêt est toujours saturée. Ce lien imposé de manière exogène entre la dette augmentée des intérêts et la valeur du collateral exclut la possibilité de faillite des entrepreneurs. Ces derniers sont toujours en mesure de rembourser leur dette, par la vente de leur collateral.

La banque centrale fixe le taux d'intérêt nominal à l'aide d'une règle de Taylor. Cette dernière est une fonction de réaction utilisée par la banque centrale afin de choisir le niveau du taux d'intérêt nominal de référence de l'économie, fonction croissante du taux d'inflation et du PIB, exprimés en écart à leur cible. L'introduction de la règle de Taylor nous permet ainsi d'analyser les mécanismes de transmission des chocs de taux d'intérêt.

\subsubsection{Mécanismes de transmission et d'amplification}

L'étalonnage des paramètres fondamentaux est fondé sur l'article de Iacoviello (2005). Nous mettons l'accent sur les effets qualitatifs des chocs agrégés ${ }^{10}$.

\section{a) L'accélérateur financier : le cas de la bulle immobilière}

Supposons que l'économie soit frappée par un choc qui engendre une hausse du prix de l'immobilier, comme cela a été observé dans les pays industrialisés dans la période antérieure à la crise de 2008. A cette fin, à l'instar de Iacoviello (2005), nous analysons les effets d'un choc exogène de demande ${ }^{11}$ en faveur des biens immobiliers (Graphique 1). La hausse de la demande pour les biens immobiliers renchérit le prix sur ce marché. Le prix des biens immobilier rapporté à celui du bien non durable augmente. L'accélérateur financier, introduit dans le modèle via la contrainte d'endettement, vient amplifier ces effets. En effet, la hausse de la demande de biens immobiliers ainsi que l'augmentation de son prix accroissent la valeur du collateral, ce qui permet aux entrepreneurs d'emprunter davantage. Cette hausse de la demande de financement est rendue possible par un accroissement de l'offre de financement,

\footnotetext{
${ }^{10}$ Nous présentons dans cette section une version simplifiée de KM, fondée sur Iacoviello (2005). Le collateral est le bien immobilier. Afin d'illustrer de manière transparente le mécanisme d'accélérateur financier, le modèle est présenté sans rigidités de prix, sans coût d'ajustement sur le capital. Cela explique la faible persistance des effets reportés dans le graphique 1: le PIB revient en quelques trimestres à son niveau de long terme. La hausse de la dette, observée immédiatement à la période de l'occurrence du choc, implique un alourdissement du service de la dette, ce qui incite l'entrepreneur à se désendetter rapidement.

${ }^{11}$ Pendant le période 1983-2007, environ la moitié de la variation des prix de l'immobilier en France peut être expliquée par des chocs de demande (IMF, 2008). Environ 5\% de la variation de la production aurait été liée aux chocs de demande de l'immobilier. Aux Etats-Unis, ces valeurs augmentent jusqu'à $60 \%$ et $23 \%$ environ. Nous considérons un choc de $1 \%$ de la demande de bien immobilier. L'ampleur des effets peut paraître faible sur le graphique 1. Toutefois, dans les données américaines, la volatilité estimée par Iacoviello (2005) s'établirait à $25 \%$.
} 
soit une hausse de l'épargne des ménages patients. Ces derniers accentuent leur effort d'épargne et consomment moins.

Etant donné que les entrepreneurs sont plus impatients, ces derniers sont incités à consommer davantage, ce qui, avec l'augmentation du stock du capital, relance la production.

De plus, hypothèse conforme aux usages en matière de prêts bancaires, les entrepreneurs qui s'endettent auprès de la banque s'engagent à rembourser leurs crédits des euros courants, et non en euros constants. La dette n'est pas indexée sur l'inflation. Cette dernière, par un effet re-distributif, amplifie les effets richesse en faveur des emprunteurs. L'augmentation de l'inflation associée au choc de demande réduit la valeur réelle de la dette des entrepreneurs, ce qui vient nourrir l'effet richesse positif en leur faveur.

\section{b) Le rôle du taux d'intérêt}

Plusieurs études empiriques récentes ont confirmé l'existence d'un lien négatif entre les niveaux des taux d'intérêts et la demande d'actifs immobiliers. Pendant la dernière décennie, les faibles niveaux de taux d'intérêt ont été associés à une hausse importante des prix ${ }^{12}$ de l'immobilier (Iacoviello et Neri, 2010). Nous analysons donc la réponse de l'économie à un choc qui réduit de manière temporaire le taux d'intérêt nominal. Une diminution du taux d'intérêt implique une baisse des coûts du service de la dette (Graphique 2). Les entrepreneurs peuvent s'endetter davantage. Cela est rendu possible par une hausse de l'offre de financement des ménages - banquiers qui accentuent leur effort d'épargne par une baisse de leur consommation. Les entrepreneurs augmentent leur stock de dette afin de consommer davantage et acheter des biens immobiliers.

Les entrepreneurs consomment davantage des biens immobiliers comme des biens non durables. Le prix de ces deux biens augmente. En l'absence de rigidité de prix, la hausse du prix des biens non durable domine à court terme celle du prix de l'immobilier, ce qui réduit le prix relatif des biens immobiliers.

\footnotetext{
${ }^{12}$ En France, pendant le période 1983-2007, l'élasticité du prix réel de l'immobilier par apport aux taux d'intérêt été environ 3,5\%, aux Etats Unis 3\%, en Angleterre 2,3\% et en Espagne 5\% (IMF, 2008).
} 
Graphique 1 : Dynamique de l'économie suite à une hausse temporaire et inattendue de $1 \%$ la demande pour les biens immobiliers. La réponse des variables est reportée en écart en \% à

l'équilibre de long terme de l'économie
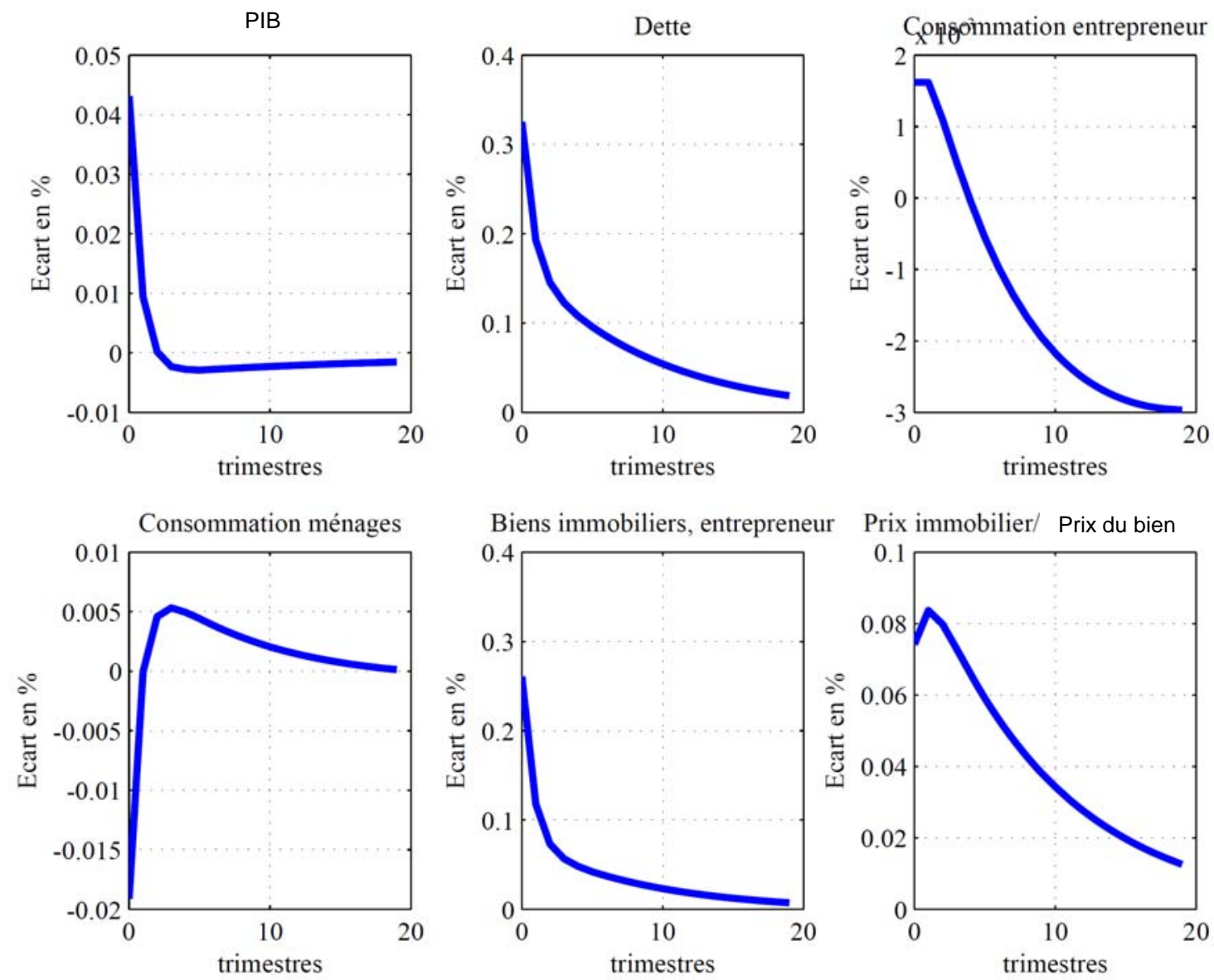

La forme en « $U$ » inversée de la dynamique de la consommation des entrepreneurs est due à un effet d'amplification retardé du choc. Ce dernier incite les agents à augmenter leur stock d'actifs immobiliers. Les entrepreneurs peuvent donc, à la période suivante, emprunter et consommer davantage. Cela est cohérent avec les faits stylisés soulignés par Bernanke et Gertler (1995) : suite à un choc monétaire temporaire, l'investissement en actifs immobiliers réagit immédiatement tandis que la réponse de la consommation de biens non - durables est retardée d'une période. Le modèle est cohérent avec une dynamique en cloche de la consommation. Bernanke et Gertler (1995) soulignent que, dans les études empiriques, même si les chocs monétaires sont temporaires, l'impact sur le revenu est persistant. Dans le modèle de KM, la persistance de la consommation est expliquée par le retard dans l'effet d'amplification sur la demande agrégée.

\section{c) Le rôle des chocs d'offre}

Dans le cas des chocs de demande, l'inflation amplifie les effets de l'accélérateur financier. En revanche, dans le cas de chocs d'offre (Graphique 3), on observe une corrélation négative entre l'inflation et la production. Cet effet modère alors ceux de l'accélérateur financier. A la suite, par exemple, d'un choc positif de productivité, le fléchissement de l'inflation renchérit la valeur de la dette en termes réels. Cet effet richesse négatif implique une chute du stock des biens immobiliers (et donc du collateral) et une baisse plus marquée de la dette. 
Graphique 2 : Dynamique de l'économie suite à une baisse temporaire et inattendue de $1 \%$ du taux d'intérêt nominal. La réponse des variables est reportée en écart en \% à l'équilibre de long terme de l'économie
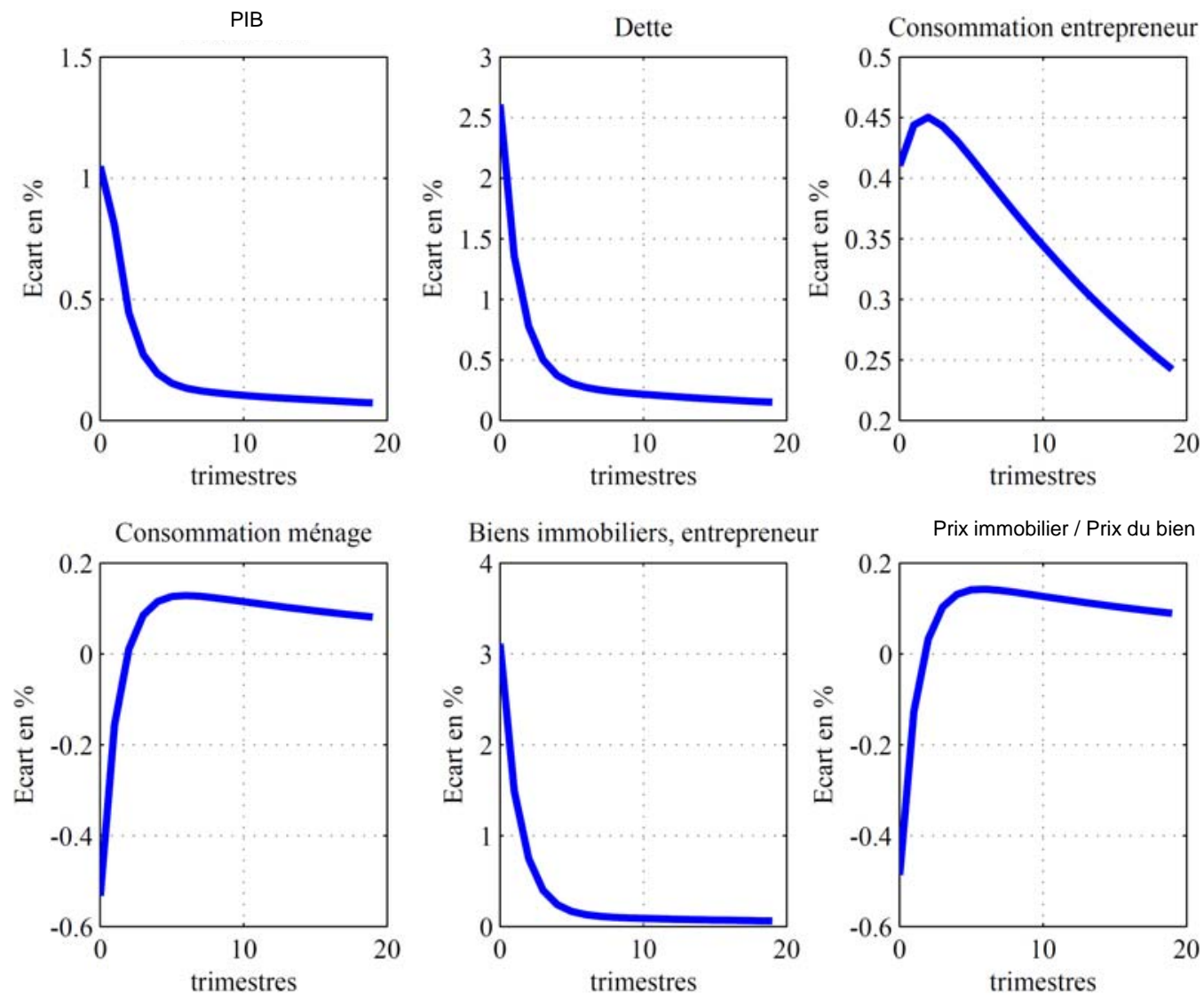

Les entrepreneurs étant plus impatients et leur utilité marginale dérivant de l'épargne (en bonds) étant inférieure à celle des banquiers, les entrepreneurs n'épargnent pas et consomment davantage. Ils ont donc un impact important sur la demande agrégée.

Afin d'illustrer le mécanisme d'amplification financière, il convient d'analyser le rôle de la contrainte de collateral. Ces effets d'accélération devraient s'accentuer avec la quantité de dette. Nous considérons donc différentes valeurs de $m$, le paramètre qui représente la fraction de la valeur des actifs immobiliers qui peut être utilisé en tant que collateral. En effet, étant donné que, à l'équilibre, la contrainte de collateral est saturée, la dette augmente avec ce paramètre. Le graphique 4 illustre le lien entre la volatilité de la production et la valeur de $m$. Notre exercice numérique repose sur la simulation de l'écart type de la production en réponse à une série standard de chocs ${ }^{13}$.

La volatilité du PIB augmente avec le paramètre $m$. La possibilité de relâcher la contrainte d'endettement favorise l'amplification des fluctuations du cycle réel. C'est là l'essence du mécanisme d'accélérateur financier. Ce dernier est donc particulièrement opérant dans des

\footnotetext{
${ }^{13}$ Nous avons introduit simultanément tous les chocs analysés séparément dans ce paragraphe : un choc de préférence, un choc de productivité et un choc qui réduit temporairement le taux d'intérêt nominal. Tous les chocs, pour ce graphique, sont caractérisés par un écart-type de $1 \%$ de façon à illustrer l'impact de tous les chocs sur la volatilité du PIB.
} 
économies endettées. Nous retrouvons ce résultat dans le modèle de BGG. Si un gouvernement souhaite stabiliser son économie (réduire la volatilité des fluctuations du PIB), il tentera de baisser de $m$. Il s'agit d'inciter les banques à être plus strictes dans l'octroi de crédits de sorte que la contrainte d'endettement des emprunteurs est plus serrée. ${ }^{14}$

Graphique 3 : Dynamique de l'économie suite à une hausse temporaire et inattendue de $1 \%$ de la productivité globale des facteurs. La réponse des variables est reportée en écart en \% à

l'équilibre de long terme de l'économie
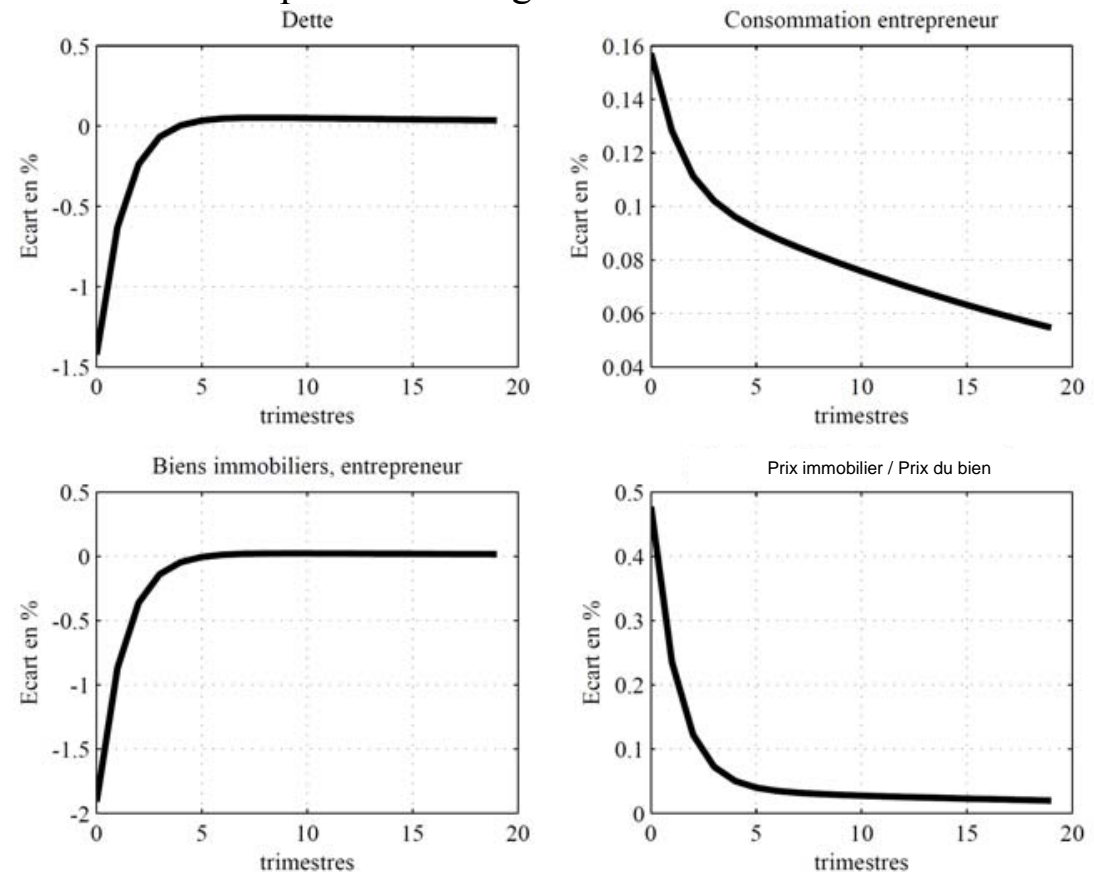

\section{Le modèle de Kiyotaki et Moore (1997) au défi de la crise}

L'article de KM s'impose comme une contribution de référence. En effet, ce travail constitue l'une des premières analyses d'équilibre général qui tient compte des effets d'amplification associés aux imperfections du marché de crédit. Ce cadre a été utilisé récemment pour expliquer les faits stylisés caractérisant la période antérieure à la crise. Notre analyse qualitative a souligné les mécanismes du modèle qui permettent de reproduire le lien négatif entre les taux d'intérêt et le boom de l'immobilier et, de manière générale, la corrélation positive entre consommation et richesse immobilière. ${ }^{15}$ De plus, elle permet d'éclairer comment le relâchement des contraintes de crédit qui a caractérisé les deux dernières décennies peut impliquer une augmentation de la volatilité agrégée et une amplification du cycle.

En revanche, ce cadre théorique recèle des limites importantes qui ne permettent pas d'analyser plusieurs mécanismes à la base de la crise actuelle.

i) Tout d'abord, ce cadre néglige le rôle spécifique de l'intermédiation financière. Les ménages agissent ici en tant que banquiers seulement parce que, à l'équilibre, ils sont créditeurs. L’intermédiation financière est en réalité inexistante.

\footnotetext{
${ }^{14}$ Cela ne signifie pas pour autant qu'une telle mesure est optimale. En effet, un endettement moindre des entrepreneurs vient limiter leur activité productive et leur consommation.

${ }^{15}$ L'ampleur de cette corrélation dépend de l'étalonnage retenu. Lorsque l'économie est caractérisée par une forte proportion d'agents très impatients, la corrélation entre consommation et richesse immobilière sera forte.
} 
ii) De plus, le modèle de KM fait abstraction de la faillite des emprunteurs. Cela est clairement en contradiction avec la hausse des taux de défaut de paiement observés lors de la crise (CAE 2008; Christiano, Motto et Rostagno, 2009).

iii) Enfin, le ratio d'endettement des entrepreneurs est constant au cours du cycle économique (égal au paramètre $m$ exogène). Cela est conforme à la pratique en cas de prêt immobilier : la banque finance une proportion fixe de la valeur du bien. Or, les crédits concernent plus largement le financement de l'ensemble de l'activité des banques comme des entreprises. Or, on observe que le ratio d'endettement des agents financiers et non financiers répond au cycle économique (Banque de France, 2009, sur données américaines et européennes). Ces fluctuations sont de nature à amplifier ou modérer l'impact des chocs macroéconomiques.

Graphique 4 : Fraction de la valeur des actifs immobiliers qui peut être utilisé en tant que collateral et volatilité du PIB. La volatilité du PIB est mesurée par l'écart-type des fluctuations du PIB prédit par le modèle. Ces valeurs sont à comparer aux volatilités observées suivantes (en \%): 0,78 pour la France; 0,7pour la zone Euro; 1.01 pour les EtatsUnis. Source : Calcul des auteurs à partir des données trimestrielles AWM et Fed de Saint Louis, 1995:1-2007:4, log du PIB par habitant, filtré par la méthode de Hodrick et Prescott (1997)

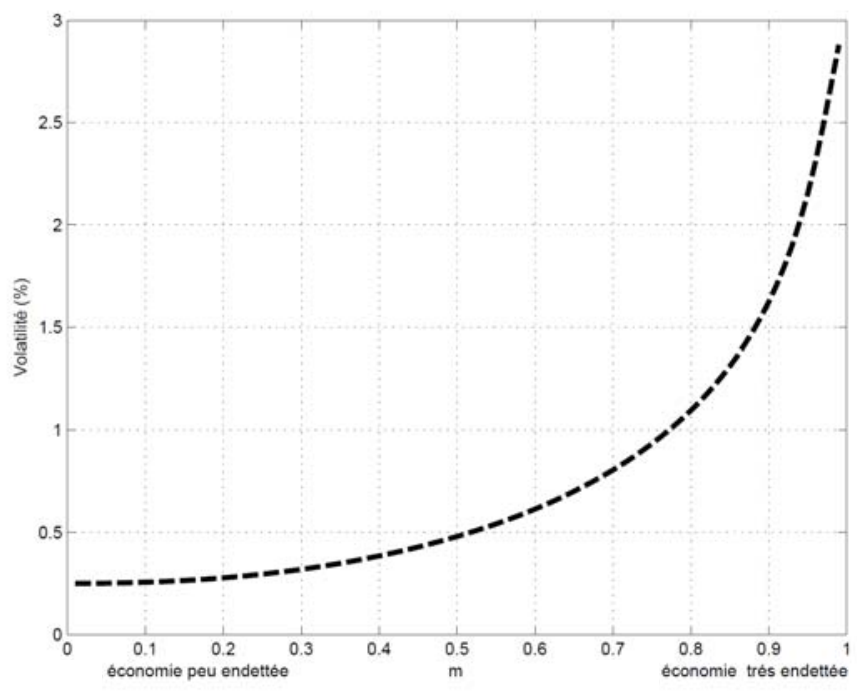

\section{2. L'accélérateur financier dans le canal large du crédit: Bernanke, Gertler et Gilchrist (1999)}

Quel est le rôle spécifique du secteur financier dans l'évolution des variables macroéconomiques ? Dans BGG, les facteurs financiers amplifient les effets de la politique monétaire par deux canaux : le premier reprend le mécanisme lié aux variations du prix des actifs financiers (à l'instar de KM) tandis que le second a trait aux fluctuations du levier.

BGG répondent aux critiques adressées au modèle de KM.

i) Il s'agit de proposer une modélisation dans laquelle les intermédiaires financiers jouent un rôle spécifique dans la relation de crédit. Les banques développent un savoir faire dans la connaissance de la solvabilité des emprunteurs et la vérification du succès des projets d'investissement pour lesquels les entrepreneurs ont sollicité un 
financement bancaire. Cette activité de gestion des crédits nécessite une rémunération, ce qui introduit un écart entre le taux d'intérêt qui rémunère les dépôts et ceux appliqués aux crédits. Ce spread de taux constitue l'élément nouveau dans la modélisation de BGG par rapport à celle de KM, cadre dans lequel le taux d'intérêt est unique.

ii) Les emprunteurs peuvent se déclarer en défaut de paiement sur leur dette, ce qui est exclu dans KM.

iii) Le ratio d'endettement des entrepreneurs est endogène. En effet, les entrepreneurs se financent auprès de la banque. Cette dernière détermine le taux d'intérêt associé au crédit en fonction de la solvabilité de l'emprunteur, résumée par sa richesse nette. Les fluctuations de la richesse nette affectent les conditions de financement et donc le ratio d'endettement.

iv) Notons que le levier de la banque est toujours égal à $100 \%$ car la banque ne se finance qu'au moyen des dépôts des ménages. L'hypothèse d'absence de capital bancaire sera dépassée dans les articles exposés dans la section 3.

A la suite d'un choc macroéconomique, le modèle de BGG prédit une réponse endogène de ces trois éléments : le spread de taux, le taux de défaut de paiement des entrepreneurs et leur levier.

\section{2. 1. Le contrat de dette}

BGG soulignent le rôle spécifique des banques dans la relation de crédit. Ils se placent dans un cadre dans lequel les demandeurs de crédit sont constitués d'un ensemble d'entrepreneurs désireux d'accroître leur stock de capital. L'investissement est financé par auto-financement (via la richesse nette) et par emprunt bancaire. Le ratio d'endettement est défini comme la part du stock de capital financé par emprunt. Les entrepreneurs, neutres au risque, subissent un choc qui leur est spécifique sur le rendement de leur capital, choc qui vient affecter leur capacité de rembourser leur dette.

Les banques subissent l'asymétrie d'information inhérente à la relation de crédit. Elles ne sont pas en mesure d'évaluer correctement la solvabilité des emprunteurs (asymétrie d'information ex-ante, avant la signature du contrat de dette), ni l'utilisation effective des fonds empruntés (asymétrie d'information ex-post, après la signature du contrat de dette), ni le rendement des projets d'investissement pour lesquels les financements sont demandés (asymétrie ex-post). Ces asymétries d'information sont de nature à réduire l'offre de crédits.

Pour remédier aux asymétries d'information ex-ante, les banques sélectionnent les demandes d'emprunt en fonction de caractéristiques de l'emprunteur, caractéristiques signalant sa capacité à rembourser. Les emprunteurs caractérisés par une solvabilité jugée médiocre se verront proposer des conditions plus strictes d'accès au crédit. Dans le modèle de BGG, le montant de la richesse nette de l'emprunteur détermine le taux d'intérêt associé à la dette bancaire. Plus l'emprunteur est riche au moment de sa demande de crédit, plus il est jugé solvable, ce qui vient modérer le taux d'intérêt associé à sa dette bancaire.

Dans le modèle de BGG, l'asymétrie d'information ex-post concerne le rendement du projet d'investissement pour lequel l'entrepreneur sollicite un financement bancaire. Les termes du contrat de dette doivent inciter l'emprunteur à déclarer sans malhonnêteté ce rendement, qu'il soit capable de rembourser sa dette ou non, et ce pour deux raisons. 
- En cas de défaut de paiement, un audit mis en œuvre par la banque auprès de l'emprunteur. Au terme de cette procédure de vérification, la banque a connaissance de la valeur du rendement du capital.

- En cas de non défaut de paiement, le remboursement de la dette est une proportion fixe du rendement du capital.

La procédure d'audit est coûteuse et répercutée par la banque sur les conditions d'accès au crédit: le taux d'intérêt sur la dette bancaire doit rémunérer la banque pour son rôle d'auditeur. En conséquence, le financement bancaire de l'investissement s'avère plus onéreux que l'autofinancement dont le coût d'opportunité est le taux d'intérêt sans risque. La différence entre les coûts associés aux deux modes de financement de l'investissement constitue la prime de financement externe.

- En l'absence d'asymétrie d'information, la prime de financement externe est nulle. Le théorème de Modigliani Miller est vérifié : le montant de l'investissement est indépendant de son mode de financement.

- En revanche, la présence d'asymétrie d'information implique la mise en place de l'audit en cas de défaut de paiement. La prime de financement externe vient renchérir le coût du financement bancaire par rapport à l'autofinancement.

Le contrat de dette dans le modèle de BGG conduit à une prime de financement externe décroissante dans la richesse nette de l'emprunteur. Un entrepreneur disposant d'une richesse nette élevée bénéficie de conditions de financement avantageuses, ce qui l'incite à s'endetter pour financer l'augmentation de son stock de capital. La taille de l'entreprise augmente grâce à cet effort d'investissement plus marqué. Toutefois, un endettement plus marqué accroît la probabilité de défaut de paiement. Cela constitue la limite à l'expansion de l'endettement de l'entreprise.

\section{2. 2. Dynamique macroéconomique dans le modèle de BGG}

BGG intègrent ce contrat de dette dans un modèle d'équilibre général permettant de quantifier l'interaction entre les facteurs financiers et les variables macroéconomiques. Une telle tâche requiert de résoudre plusieurs difficultés exposées en annexe A.

\section{a) Le modèle macroéconomique}

Le modèle macroéconomique spécifie le comportement des ménages et la politique monétaire. L'épargne des ménages déposée auprès des banques est rémunérée au taux d'intérêt nominal certain. L'inflation peut donc éroder la valeur réelle des dépôts. Après avoir effectué le choix du montant de son dépôt bancaire, le ménage est dans l'impossibilité de le récupérer avant la période suivante. Cela exclut la possibilité de «bank run » dans la période. Cette hypothèse forte est abandonnée dans les travaux de Angeloni et Faia (2010) présentés dans la section 3. 2. 3. b)

Les dépôts sont ensuite utilisés par la banque pour distribuer des crédits aux entrepreneurs à un taux d'intérêt supérieur au taux d'intérêt certain. Le profit de la banque est nul : l'ensemble des gains sur les crédits distribués (sur lesquels il n'y a pas de défaut de paiement) permet de servir aux ménages le taux d'intérêt certain sur leur dépôt bancaire. Dans le modèle de BGG, l'égalité entre le rendement sur les actifs risqués et la rémunération certaine sur les dépôts suggère que la banque commerciale est capable d'opérer sur la diversité des crédits distribués une diversification des risques suffisante pour garantir un taux d'intérêt sans risque sur les fonds qui lui sont confiés. De plus, la faillite bancaire est exclue dans le modèle de BGG. Ces 
caractéristiques font du modèle de BGG un cadre moins pertinent pour analyser la crise financière actuelle. Cette limite sera dépassée dans la section 3.

BGG analysent les canaux de transmission de la politique monétaire. La banque centrale choisit le taux d'intérêt nominal certain selon une règle de Taylor. A l'instar du modèle de $\mathrm{KM}$, l'inflation, par son effet redistributif en faveur des emprunteurs, affecte les mécanismes intervenant dans l'accélérateur financier.

\section{b) La dynamique macroéconomique}

Une conjoncture favorable, associée par exemple à une progression non anticipée de la productivité, vient augmenter la demande d'investissement (Graphique 5). Cette dernière accroît le prix du capital, donc le montant de la richesse nette, ce qui améliore les termes de financement des entrepreneurs : la prime de financement externe baisse. Ce mécanisme est similaire à l'accélérateur financier de KM.

Le seuil de productivité du choc spécifique à l'entrepreneur en dessous duquel les entreprises se déclarent en défaut de paiement baisse instantanément $\left(\omega^{*}\right)$, ce qui correspond à une baisse de la probabilité de défaut de paiement. Les entrepreneurs profitent des conditions financières favorables pour investir davantage, ce qui vient amplifier l'effet initial de la hausse de la productivité. Le boom de l'investissement nourrit une hausse du stock de capital, de la production et de l'emploi. Un cercle vertueux est enclenché dans l'économie.

Les effets du choc agrégé favorable s'estompent progressivement car la hausse du ratio d'endettement accroît la probabilité de faillite (hausse de $\omega^{*}$ ). Les conditions de financement deviennent moins favorables : la prime de financement externe augmente. De plus, la hausse graduelle du stock de capital réduit sa productivité, ce qui explique le ralentissement de l'investissement observé après le boom initial.

Le choc d'offre réduit instantanément l'inflation. A l'instar du modèle de KM, le fléchissement de l'inflation redistribue la richesse en défaveur des entrepreneurs. Cet effet limite l'expansion de la richesse nette au cœur de l'accélérateur financier.

Sur le graphique 5, nous observons une hausse concomitante de l'autofinancement (par l'expansion de la richesse nette des entrepreneurs) et de la dette (qui finance une expansion de l'investissement), de sorte que, a priori, la part relative de la dette dans le financement peut augmenter ou baisser. En réalité, le ratio dette / autofinancement augmente. Cela est visible par le fléchissement de la prime de financement externe qui, dans le modèle, est une fonction décroissante du levier. Plus la part de la dette augmente dans le financement, plus la banque juge peu solvable l'emprunteur, ce qui l'incite à relever le taux d'intérêt sur les crédits. Le levier des entrepreneurs est procyclique dans BGG.

Ces mécanismes économiques sont également à l'œuvre pour tous les autres chocs macroéconomiques. Dans le cas d'une politique monétaire expansionniste non anticipée (graphique 6), le fléchissement du taux d'intérêt nominal certain réduit la prime de financement externe. Les entrepreneurs profitent de ces conditions favorables de financement pour accentuer leur effort d'investissement, ce qui vient renchérir le prix du capital. Cet accroissement non anticipé du prix des actifs vient augmenter la richesse nette des entrepreneurs, ce qui nourrit une nouvelle baisse de la prime de financement externe, venant amplifier l'effet initial de la politique monétaire. 
Graphique 5 : Réponse de l'économie à une hausse inattendue et temporaire de $1 \%$ de la productivité globale des facteurs. La réponse des variables est reportée en écart en \% à l'équilibre de long terme de l'économie
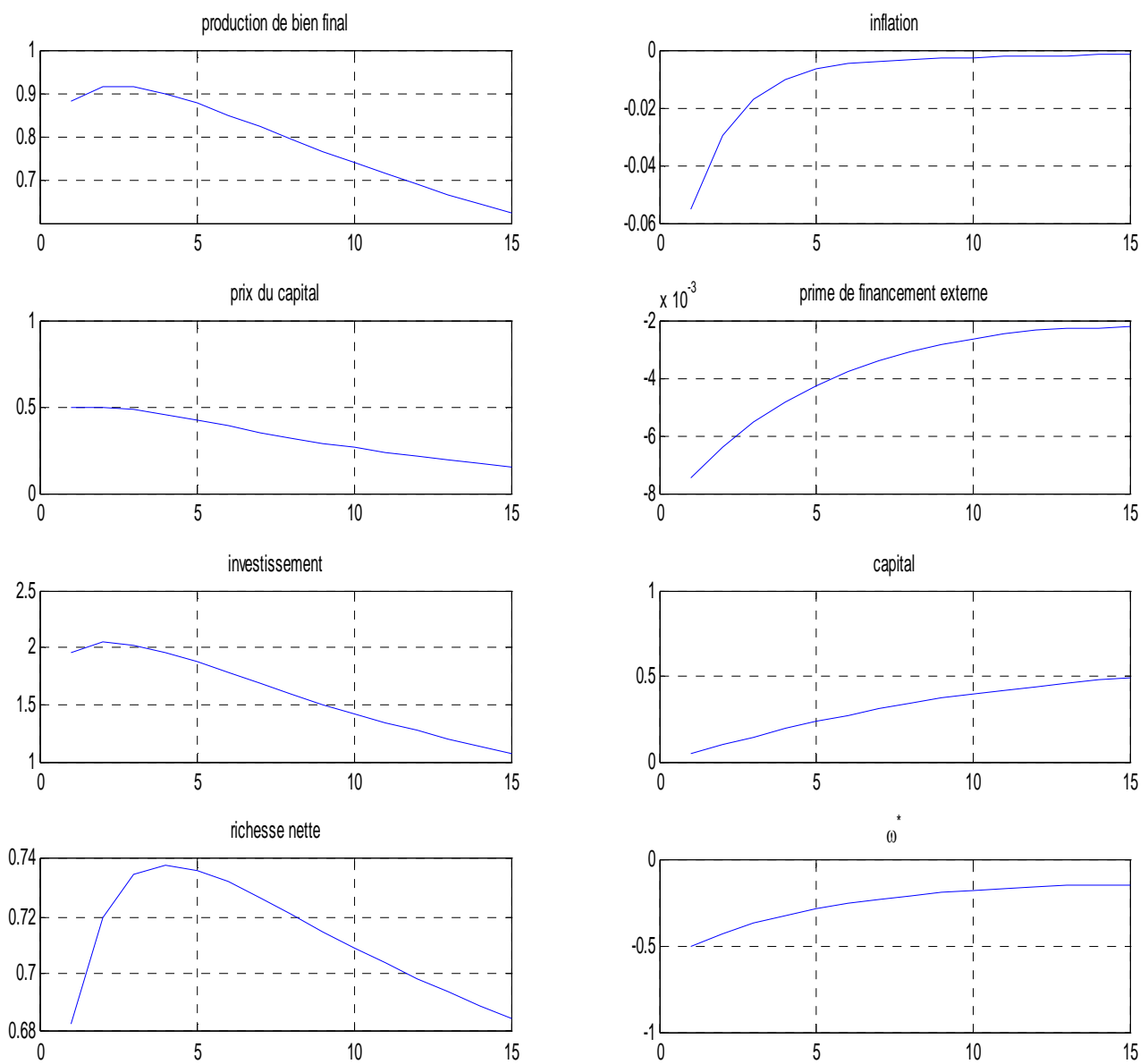

Enfin, l'inflation associée à la politique monétaire expansionniste redistribue la richesse en faveur des entrepreneurs. Cet effet vient renforcer les effets de l'accélérateur financier dans le cas du choc monétaire alors qu'il avait tendance à modérer ses effets dans le cas du choc d'offre.

Ayant simulé leur modèle étalonné sur données américaines, BGG montrent que la réponse instantanée de l'activité est supérieure de $50 \%$ à celle observée dans un modèle dépourvu d'accélérateur financier. De plus, conformément aux évolutions observées aux Etats-Unis, l'effet de la politique monétaire sur la production perdure alors même que le taux d'intérêt nominal est revenu à son niveau initial.

Quel que soit le choc envisagé, nous observons, dans le modèle de BGG, une prime de financement externe contracyclique et des prix des actifs procycliques. Cela est conforme aux fluctuations observées aux Etats-Unis comme dans la Zone Euro (Christiano, Motto et Rostagno, 2010). Au-delà de l'évolution de ces variables financières, la pertinence empirique du modèle de BGG a été évaluée au regard de ses prédictions macroéconomiques à la suite d'une politique monétaire (Bernanke et Gertler, 1995). Les estimations structurelles du modèle d'accélérateur financier soulignent également la pertinence de ce mécanisme aux Etats-Unis comme dans les pays européens (Christensen et Dib, 2008 ; Christiano, Motto et 
Rostagno, 2010 ; Gelain, 2010 ; De Graeve 2008 ; Queijo von Heideken 2008). L'ampleur des effets de l'accélérateur financier est réglée par le ratio d'endettement de l'économie. Le cadre d'analyse est donc particulièrement pertinent dans les économies anglo-saxonnes caractérisées par des taux d'endettement de 200\% du PIB (140\% du PIB) pour les agents privés au Royaume-Uni (aux Etats-Unis respectivement) en 2008 alors que le ratio s'établit autour de 120\% du PIB pour la Zone Euro (Blot et Timbeau, 2009 et CAE 2008). Enfin, les études microéconométriques sur données d'entreprises confirment la pertinence du mécanisme d'accélérateur financier en France, dans la zone euro et aux Etats-Unis (Chatelain et al., 2003a et 2003b ; Kannan, 2010).

Graphique 6 : Réponse de l'économie à une baisse inattendue de $1 \%$ du taux d'intérêt de la banque centrale. La réponse des variables est reportée en écart en \% à l'équilibre de long terme de l'économie
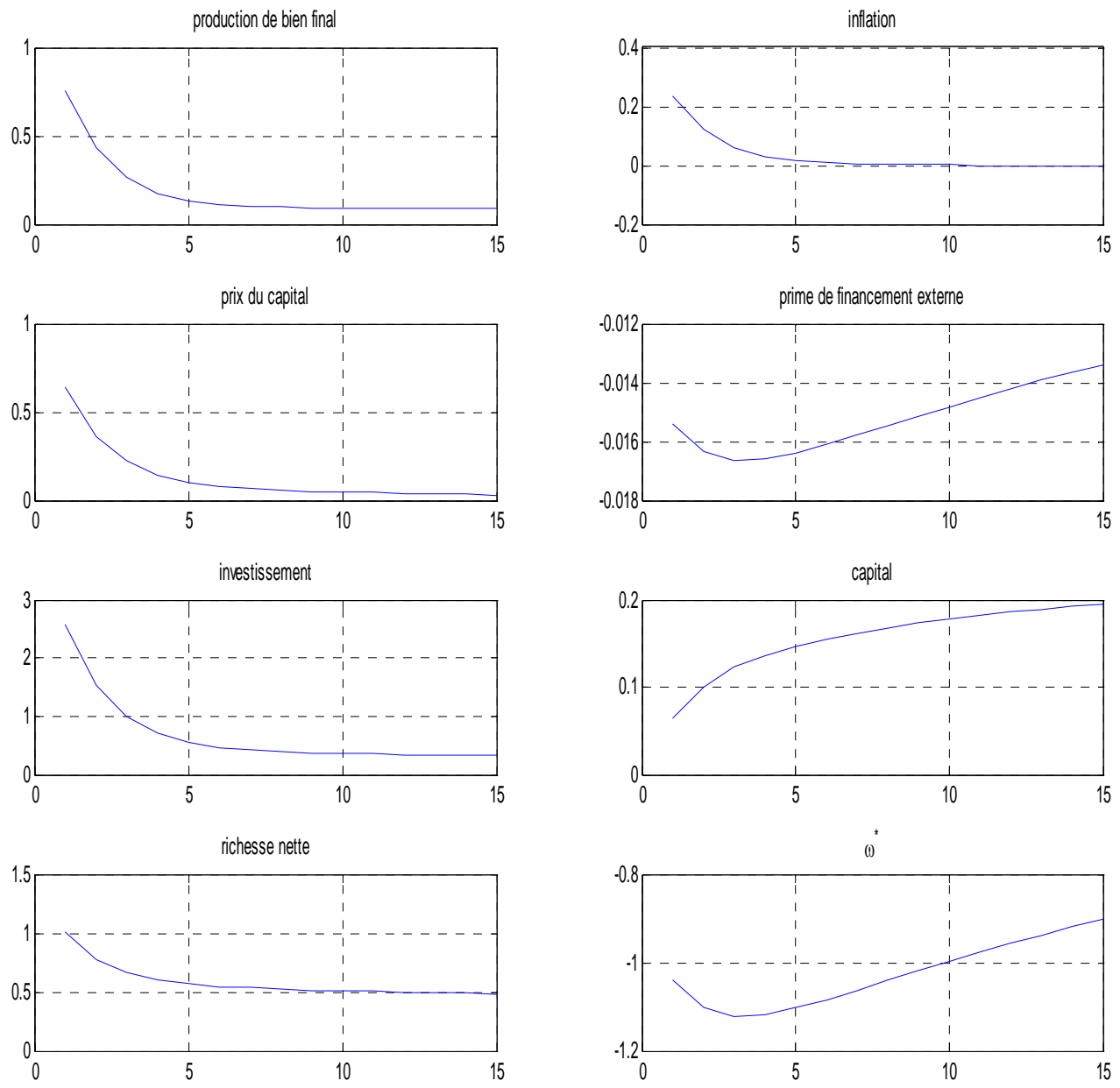

La crise financière de 2007 - 2008 vient-elle remettre en cause la pertinence empirique du modèle de BGG ? Christiano, Motto et Rostagno (2010) montrent qu'un modèle BGG augmenté de chocs financiers rend compte de la dynamique macroéconomique observée en zone Euro comme aux Etats-Unis entre 1985 et 2008. Toutefois, les événements de la crise invitent à dépasser le modèle BGG. En effet, des économistes (dont Rajan, 2005) ont attribué la responsabilité de la crise à la prise de risque excessive des intermédiaires financiers. Cela 
suggère de modéliser une banque qui choisit son exposition au risque dans la composition de son actif ou de son passif, ce qui n'est pas le cas dans BGG, modèle dans lequel la banque est qualifiée de voile en raison de l'absence de décision qui caractérise cet agent dans l'économie.

Dans BGG, la parfaite diversification des risques sur les crédits octroyés aux entrepreneurs ne laisse aucun rôle à la banque. Cette dernière est caractérisée par un rôle passif dans la distribution des crédits puisque la politique d'offre de crédit est conduite simplement par l'ajustement systématique de la prime de financement externe des entrepreneurs en fonction de l'évolution de leur richesse nette. Dans BGG, la banque ne prend aucune décision concernant la taille ou la composition de son passif : ce dernier est exclusivement composé des dépôts des ménages. Ce sont ces derniers qui déterminent l'ampleur des ressources dont dispose la banque dans la distribution de crédit. Or, les banques disposent en réalité de capitaux propres. Les difficultés de refinancement des intermédiaires financiers ont constitués les éléments déclencheurs de la crise.

\section{La crise financière: un défi pour l'analyse macroéconomique}

La crise actuelle invite à dépasser les deux modèles fondateurs. En effet, de nouveaux défis se présentent (section 3.1) auxquels les développements récents (section 3.2.) tentent de répondre.

\subsection{Quels défis?}

\section{a) Un défi quantitatif}

La crise constitue un défi quantitatif car

- Elle affecte l'ensemble de l'économie : la crise financière affecte d'abord les banques avant de frapper l'économie réelle. La crise repose la question du passage d'une crise financière à une récession économique.

- Elle se distingue par l'ampleur de ses effets : le prix des actifs financiers et les spreads de taux ont connu de fortes variations, aussi brutales que persistantes (CAE 2008); ce qui a donné lieu à des conséquences macroéconomiques dont l'ampleur a conduit les économistes à qualifier la dernière crise de «Grande Récession ». La période 2007-2009 aux Etats-Unis a été déclarée comme la crise la plus longue depuis 1945 d'après le NBER, avec 18 mois de ralentissement de l'activité contre 11 mois en moyenne depuis l'aprèsguerre. Elsby et al. (2010) montrent que la dernière crise se distingue aux Etats-Unis par une dégradation sans précédent de la sortie de chômage.

Les modèles visant à capter les effets de la crise doivent donc

- prendre en considération de nouvelles sources de perturbations dans l'économie, ayant pour origine le secteur financier; au-delà des chocs usuels (technologie, dépenses publiques, ...)

- mettre l'accent sur des effets d'amplifications du secteur financier à l'économie réelle, audelà de ceux inhérents à l'accélérateur financier.

o Une question se pose alors non seulement sur la capacité des modèles à reproduire les fortes variations du prix des actifs et des spreads de taux observés sur les marchés financiers mais également à relier ces fortes variations au ralentissement macroéconomique marqué des pays de l'OCDE. 
o Il s'agit également de préciser quels spreads de taux sont pertinents dans cette analyse de la transmission du secteur financier à l'économie réelle.

- Dans BGG, le spread de taux désigne le différentiel entre le taux d'intérêt payé par les entreprises qui s'endettent et le taux d'intérêt certain.

- Gertler (2010) rappelle que, à la suite de l'épisode Lehman Brothers, en septembre 2008, le spread de taux pour les agents non financiers aux EtatsUnis a augmenté de 500 points de base (contre 100 points de base lors de la crise de 1982) et de 800 points de base sur le marché interbancaire (contre 50 points de base lors de la crise de 1982). Ce constat suggère qu'une bonne compréhension de la crise passe par une modélisation des tensions sur les possibilités de financement des intermédiaires financiers. ${ }^{16}$

- comprendre la dynamique non linéaire qui a caractérisé la crise : une phase d'embellie, soudainement interrompue par un dégonflement du prix des actifs financiers et une récession. («Boom-bust cycle » ou « Sudden Stop »)

\section{b) Evolution du secteur financier}

L'essor des marchés financiers observé aux Etats-Unis et en Europe depuis la fin des années 70 semblait sonner le glas des établissements de crédits. Capelle-Blancard et CouppeySoubeyran (2003) et le rapport du CAE (2011) rappellent qu'il n'en n'est rien. Les établissements de crédit jouent encore un rôle majeur dans le financement de l'économie. Ce financement passe désormais autant par l'octroi de crédits que par l'acquisition de titres financiers. Ces études montrent que l'accent des MEGIS sur la modélisation de la banque reste justifié. La crise récente appelle à une nuancer cette constatation.

La faillite de Lehman Brothers en septembre 2008 est représentative de l'évolution du secteur financier depuis les contributions de KM et BGG. Lehman Brothers est un intermédiaire financier du secteur bancaire parallèle ("shadow banking»), appelé ainsi car il s'agit d'institutions financières qui ne collectent pas des dépôts auprès des ménages (ces derniers relevant de la législation sur l'assurance des dépôts), échappant donc à la réglementation bancaire. Ces intervenants (hedge funds, banques d'investissement, véhicules financiers spécifiques) s'endettent à court terme auprès des marchés financiers (en émettant des titres de dette de court terme) pour acquérir des titres risqués. Brender et Pisani (2010) rappellent que la prise de risque est particulièrement visible dans l'évolution du bilan des acteurs de la banque parallèle par un déséquilibre accru entre structure du passif sans risque et structure de l'actif plus risqué. Par ces acquisitions de titres émis par des agents financiers ou non financiers, les acteurs du "shadow banking» participent au financement de l'économie. Adrian, Ashcraft, Boesky et Pozsar (2010), Adrian et Shin (2010) rappellent le poids du "shadow banking" dans le financement de l'économie américaine, qui, avant la crise, rivalise, à hauteur égale, avec celui des banques commerciales traditionnelles. En revanche, en zone euro, les prêts des banques traditionnelles sont demeurés de loin la principale source de financement des agents non financiers entre 2000 et 2008 (BCE, 2009).

\footnotetext{
${ }^{16}$ Au-delà de ces interrogations, d'autres auteurs s'interrogent sur l'attention portée au spread de taux. Les travaux de Chari, Christiano et Kehoe (2008) invitent à ré-examiner l'ampleur de la crise financière en arguant, en particulier, que l'accent sur les spreads de taux n'est pas judicieux dans une période marquée par des taux d'intérêt sans risque très faibles. Dans un contexte financier dans lequel le service de la dette reste modéré, la mesure du risque par le spread de taux peut être biaisée. Gilchrist et Zakrajek (2011) soulignent également que le spread de taux ne constitue un bon indicateur avancé de l'activité économique que s'il tient compte des fluctuations de la relation entre le risque de défaut des entreprises et le spread.
} 
Les titres acquis par les acteurs de la banque parallèle sont des créances titrisées par les banques commerciales traditionnelles. La titrisation désigne l'opération qui consiste à transformer un actif illiquide, comme un encours de prêts, en titres négociables (CAE 2008 par exemple fournit une présentation claire de ce procédé). Un crédit octroyé par la banque traditionnelle peut donc être cédée à un tiers sur le marché des créances titrisées. Ce faisant, les encours de prêts titrisés sont sortis des bilans des banques commerciales pour entrer dans celui des «shadow banks ». L'essor du « shadow banking » est alimenté par la titrisation. Le modèle bancaire fondé sur le « originate and hold» (initier le crédit, le suivre en le gardant dans le bilan) laisse place au " originate, repackage and sell » (initier le crédit, le titriser et le vendre). Une telle évolution incite les banques à une sélection moins rigoureuse dans sa distribution de crédit. Les banques qui ont recours à la titrisation s'engagent à en assurer la liquidité si le marché s'assèche (back up lines). Les créances titrisées sont restructurées (rassemblées dans des portefeuilles) avant d'être négociées ce qui accroît leur complexité et leur opacité. L'appréciation du risque sur ces titres a été laissée aux agences de notation. L'absence d'information claire sur le risque réel associé à ces titres a joué un rôle central dans la crise financière.

\section{c) Les politiques économiques}

Face à cette crise financière majeure, les banques centrales ont procédé à une baisse des taux directeurs. Toutefois, cette politique monétaire conventionnelle s'est heurtée à un taux plancher (zero interest rate bound). Les taux directeurs étant très bas, les banques centrales ont été dans l'impossibilité de les baisser davantage. Des politiques monétaires non conventionnelles ont alors été mises en œuvre afin de juguler la crise.

Plusieurs questions se posent dans la mise en œuvre des politiques non conventionnelles. La première concerne l'efficacité relative des différents volets qui la composent: "quantitative easing " (expansion de la masse monétaire, donc hausse de la taille du bilan de la banque centrale) versus "credit easing » (modification de la composition du bilan de la banque centrale). Dans le dispositif " credit easing », quelle mesure serait la plus efficace (octroi de crédit aux agents non financiers, direct lending; aux banques commerciales, discount window lending; ou dans la prise de participation dans le capital des banques commerciales, equity injection)?

De plus, les questions relatives à l'organisation de la fin de ces dispositifs se posent avec acuité (« Exit strategy »). Comment organiser l'extinction des mesures d'urgence sans freiner le retour à la croissance? L'analyse d'une telle question nécessite l'examen d'une coordination éventuelle entre la politique monétaire et d'autres politiques économiques telles que la politique budgétaire.

Enfin, au-delà de la gestion de la crise, se pose la question de savoir si la banque centrale doit être responsable de la stabilité financière. Il faut d'abord savoir ce que l'on entend par stabilité financière. Galati et Moessner (2011) rappellent qu'elle est multi-dimensionnelle et qu'il convient de déterminer quels aspects de la stabilité financière relève de la banque centrale. Il convient de déterminer si, en ce domaine, le principe de séparation s'applique, auquel cas la banque centrale conserverait son objectif de stabilité des prix et laisserait à un organisme indépendant (éventuellement transnational) la responsabilité de la stabilité financière. 


\subsection{L'introduction de l'intermédiation financière dans les modèles macro-économiques : apports et insuffisances}

\section{2. 1. Curdia et Woodford (2010)}

\section{a) le modèle}

Les principaux éléments du modèle sont les suivants :

- des agents hétérogènes : L'économie étudiée par CW est peuplée d'agents hétérogènes caractérisés par différentes utilités marginales de la consommation. Le premier type d'agents (qui seront, à l'équilibre les emprunteurs) valorise davantage l'utilité marginale de la consommation que le second type d'agents (qui, à l'équilibre, disposeront d'une épargne). Les ménages impatients sont toujours en mesure de rembourser leur dette. L'annexe $\mathrm{B}$ présente les hypothèses supplémentaires afférentes à cette hétérogénéité des agents, hypothèses destinées à simplifier le calcul de l'équilibre général du modèle.

- le rôle de la banque commerciale: les ménages disposant d'une épargne sont obligés de passer par la banque pour déposer leurs avoirs. Ils ne peuvent en aucun cas avoir une relation directe avec les emprunteurs. Dans le cadre de CW, les taux d'intérêt débiteur et créditeur ne sont pas égaux. Le spread est la somme de deux coûts :

o L'activité financière de création du crédit implique des coûts réels liés à la gestion des dossiers de prêt. La fonction de coûts opérationnels est croissante avec le volume de crédits. La présence de ces coûts opérationnels justifie le choix d'un taux d'intérêt sur les crédits distribués supérieur au taux rémunérant les dépôts bancaires.

o CW supposent également que la banque est incapable de distinguer les emprunteurs solvables des emprunteurs insolvables, ce qui l'inciterait à pratiquer un taux d'intérêt élevé pour tous les emprunteurs. Néanmoins, même si, faute d'information, la banque ne parvient pas à distinguer les emprunteurs, elle est capable de prévoir la fraction des emprunts qui ne sera pas remboursée sur l'ensemble de son portefeuille de crédits distribués. Cette fonction, qui représente les pertes associées aux emprunts non remboursés, est également croissante avec le volume de crédits. Cette hypothèse ad hoc reprend l'idée que l'existence de risque de défaut de paiement augmente le coût de l'intermédiation. Les intermédiaires financières maximisent les profits associés à leur activité et choisissent la quantité optimale de fonds à prêter. Ils prennent comme donné le taux d'intérêt payé aux déposants ; ce dernier est fixé par la politique monétaire. Soulignons que CW (2010) proposent un modèle dans lequel la banque est un agent qui optimise son bilan en choisissant sa taille et sa composition.

- La banque choisit le montant de ses crédits distribués compte tenu de ces 2 types de coûts. Elle choisit également le montant de ses réserves auprès de la banque centrale, ces dernières étant rémunérées à un taux d'intérêt certain choisi par la banque centrale. CW supposent également que les coûts opérationnels de la banque croissent non seulement en fonction de l'ampleur de l'activité de distribution de prêts mais également en fonction des réserves. Cette hypothèse aura son importance lors de la discussion sur la politique monétaire optimale.

Bilan de l'intermédiaire financier dans CW (2010)

\begin{tabular}{|c|c|}
\hline Actif & Passif \\
\hline $\begin{array}{l}\text { - Réserves auprès de la banque centrale } \\
\text { (rémunérées au taux sans risque } i_{t}^{m} \text { ) }\end{array}$ & $\begin{array}{l}\text { Dépôts des ménages } \\
\text { (rémunérés au taux sans risque } i_{t}^{d} \text { ) }\end{array}$ \\
\hline
\end{tabular}


- Crédits distribués (rémunérés au taux sans risque $\left.i_{t}^{b}\right)$

- Le rôle de la banque centrale : les moyens d'action de la banque centrale sont au nombre de 3

o Le choix d'intérêt certain qui rémunère les dépôts de la banque commerciale $i_{t}^{d}$

o La rémunération des réserves $i_{t}^{m}$

o Un prêt direct aux agents non financiers. CW font l'hypothèse que la banque centrale est moins efficace que la banque commerciale dans la distribution de crédit de sorte que le coût lié à l'insolvabilité de certains emprunteurs est plus élevé pour la banque centrale, pour un même volume de crédits. Cette hypothèse d'efficacité moindre de la banque centrale dans l'offre de crédit sera également retenue dans d'autres contributions et est rendue nécessaire pour expliquer que, à l'équilibre et en l'absence de chocs majeurs, le modèle prédit que la banque centrale ne procède pas à un financement direct des agents privés non financiers.

Bilan de la banque centrale dans CW (2010)

\begin{tabular}{|l|l|}
\hline Actif & \multicolumn{1}{|c|}{ Passif } \\
\hline - Prêt direct aux agents non financiers & $\begin{array}{l}\text { Réserves de l'intermédiaire financier } \\
\text { - Démunérées au taux sans risque } i_{t}^{m} \text { ) } \\
\text { risque } i_{t}^{d} \text { ) }\end{array}$
\end{tabular}

\section{b) la dynamique macroéconomique}

A chaque période, les agents font leurs choix d'épargne, reçoivent les profits de l'intermédiation financière, les revenus du travail ainsi que les profits des firmes qui produisent le bien final dans un cadre de concurrence monopolistique avec rigidités de prix. Les agents épargnants ont également accès aux titres de la dette publique ${ }^{17}$. L'offre du travail, dépend du type de l'agent. CW adoptent un cadre simplifié, dépourvu de capital.

L'analyse de la transmission des chocs montre que le degré auquel les spreads répondent à une variation de l'offre d'intermédiation financière joue un rôle important, en particulier pour les chocs qui engendrent une modification de la dette privée. Dans le cas, par exemple, d'un choc technologique, le taux d'intérêt sur les dépôts baisse tandis que le modèle prédit une augmentation du crédit. Cette dernière alourdit le coût de fonctionnement de la banque, ce qui engendre une augmentation du spreads. Ce dernier a finalement un impact négatif sur la demande agrégée. La procyclicité des spreads obtenue dans le modèle de CW est toutefois contraire aux données américaines ou européennes (Christiano, Motto et Rostagno, 2010). ${ }^{18}$ La contracyclicité du spread n'est obtenue qu'à la suite d'un choc financier. L'effet de la crise serait capté dans le modèle par un choc qui pousse à la hausse les coûts opérationnels de la banque dans sa distribution de crédits. Le modèle prédit donc une chute de l'offre de prêts et une hausse du spread.

\footnotetext{
${ }^{17}$ A cause des frictions financières de ce cadre, l'équivalence Ricardienne n'est pas vérifiée et une augmentation de la dette publique a un effet de crowding out sur la dette privée. Comme dans le cadre de Gertler et Karadi (2011), la politique de crédit/endettement du gouvernement affecte les mécanismes de transmission entraînés par les intermédiaires financiers.

${ }^{18}$ Faia et Monacelli (2005) comparent la cyclicité des spreads dans les modèles de Carlstrom et Fuerst (1997) et le modèle BGG. Ils rappellent que le spread est contracyclique dans BGG et procyclique dans Carlstrom et Fuerst (1997).
} 
L'apport de CW est double :

- Tout d'abord, la banque est désormais un agent qui optimise son bilan. Toutefois, le risque de défaut de paiement, qui constitue la source principale de l'ampleur et la persistance des spreads de taux, n'est présent dans le modèle de CW que de façon ad hoc, par les coûts liés à la distribution de crédit et le montant des réserves. La notion de risque ou de défaut de paiement, aspect essentiel de la crise financière, n'est pas au centre de la modélisation de CW.

- De plus, dans la lignée des travaux de KM, CW mettent l'accent sur l'hétérogénéité des agents. Certains agents sont si impatients qu'ils sont disposés à emprunter à un taux d'intérêt plus élevé que les autres agents. Cette disparité dans l'accès au financement constitue un élément fondamental de la crise financière, dont l'origine réside dans l'octroi de crédit à des ménages qui n'offrent pas les garanties suffisantes pour bénéficier des conditions de financement les plus avantageuses (subprimes). Cela répond également aux études empiriques qui montrent le lien entre la proportion des ménages endettés et la hausse du taux de chômage aux Etats-Unis (Mian et Su, 2009). Toutefois, CW, comme $\mathrm{KM}$, considèrent que la proportion d'agents contraints financièrement dans l'économie reste exogène, donc, indépendante des conditions économiques. Cette simplification technique est dépassée dans le cadre des modèles à agents hétérogènes ${ }^{19}$.

\section{2. 2. Le canal du capital bancaire}

Les études empiriques montrent que la composition du passif des banques affecte leur politique d'offre de crédit. Levieuge (2005) et Mésonier (2005) en résument les principaux résultats en rappelant i) que l'impact de la mise en place d'un seuil réglementaire de fonds propres dans le secteur bancaire (Bâle I) a pesé sur la croissance des crédits au début des années 90 aux Etats-Unis et en Europe et ii) sur données individuelles, les banques faiblement capitalisées sont moins généreuses en termes d'offre de crédit (par une réduction de la distribution de prêts ou d'un renchérissement du taux d'intérêt débiteur). Le " capital crunch » des établissement de crédit précèderait le « credit crunch ». La clé de compréhension de cette relation entre capital bancaire et offre de crédit réside dans les contraintes de financement auxquelles sont soumises les banques. Leur richesse nette (leurs capitaux propres) détermine leurs conditions de financement. Le canal du capital bancaire constitue une extension de l'accélérateur financier au secteur bancaire : une chute du prix des actifs financiers réduit la richesse nette des banques (via une perte sur son portefeuille d'actifs par exemple qui vient peser sur son résultat et donc son capital). La banque peut alors soit augmenter son capital, soit réduire son offre de fonds. Cette dernière option semble la plus rapide à mettre en œuvre car la levée de capitaux propres peut s'avérer coûteuse pour une banque peu capitalisée dans un contexte financier dégradé.

\section{a) L'accélérateur financier dans le secteur bancaire}

L'accélérateur financier provient de l'introduction de la procyclicité du bilan bancaire. Telle est l'origine de l'amplification des effets macroéconomiques. La nature et l'origine de cette procyclicité dépendent des modélisations retenues.

Les modèles intégrant le canal du capital bancaire sont caractérisés par un double problème d'agence.

\footnotetext{
${ }^{19}$ Pour une présentation succincte de ce type de modèles, voir The Economist, « Agents of change », July 2010.
} 
o Le premier, présent dans BGG, concerne la relation de crédit entre la banque (pourvoyeur de fonds) et les entrepreneurs (emprunteurs).

o Le second, spécifique au canal du capital bancaire, concerne la relation entre les ménages (pourvoyeurs de fonds) et la banque (emprunteur).

L'aléa moral qui caractérise la relation d'endettement de la banque auprès des ménages implique que le passif de la banque ne peut se composer uniquement des dépôts. Le passif bancaire doit inclure des capitaux propres. En conséquence, les éventuelles pertes enregistrées à l'actif de la banque seront partagées entre ménages et la banque, ce qui incite cette dernière à une bonne gestion dans sa distribution de crédit. Le sachant, les ménages sont disposés à confier leurs fonds à la banque. L'exigence de capital bancaire répond, dans ce cadre, à une logique économique (Gertler et Karadi, 2011; Meh et Moran, 2010 ; Levieuge, 2009). ${ }^{20}$ Des modèles ont également intégré l'exigence de capital inhérente aux ratios de solvabilité du type ratio Cooke et ratio de Bâle. On dit alors que la présence de fonds propres répond à une logique réglementaire (Van den Heuvel, 2002).

Pour aller au-delà des revues de la littérature proposées par Levieuge (2005) et Mésonier (2005), nous mettrons l'accent sur les contributions récentes de Meh et Moran (2010) (ciaprès MM) et Gertler et Karadi (2011) (ci-après GKr) de façon à souligner la pertinence de ce mécanisme dans la crise actuelle.

$\mathrm{MM}$ et $\mathrm{GKr}$ étudient la dynamique macroéconomique obtenue dans un modèle intégrant le canal du capital bancaire, dans lequel l'exigence de fonds propres répond à une logique économique. Dans les deux cas, la banque utilise ces ressources pour investir dans des actifs risqués

- Dans MM, l'actif de la banque est uniquement composé de prêts aux entrepreneurs qui peuvent faire faillite

- tandis que, dans GKr, la banque utilise ses ressources pour acheter des actifs risqués (actions) émis par les entrepreneurs afin de financer leurs projets d'investissement.

Une chute des ressources bancaires aura donc pour conséquence dans MM un assèchement du crédit (« credit crunch ») et, dans GKr, une vente des actifs risqués (« firesale »).

\begin{tabular}{|l|l|}
\multicolumn{2}{|c|}{ Bilan de l'intermédiaire financier dans MM (2010) et $\mathrm{GKr}(2011)$} \\
\hline Actif & - Dépôts des ménages \\
- Crédits distribués (MM) & - Fonds propres \\
- Actions (GKr) & \\
\hline
\end{tabular}

\footnotetext{
${ }^{20}$ Nous retrouvons dans ces modèles l'idée de transfert de risque (« risk-shifting ») soulignée par Allen et Gale (2000). Le « risk-shifting » se développe dans la situation suivante :

o La banque s'endette à court terme auprès des ménages pour investir dans des projets risqués (les crédits distribués) et elle dispose de la possibilité de ne pas rembourser pas sa dette en cas de chute des rendements sur les projets risqués.

o En cas de succès sur les projets risqués, la banque rembourse sa dette augmentée du paiement d'intérêt et conserve le reste.

Dans une telle situation, Allen et Gale (2000) soulignent que la banque a une préférence pour le risque. Les modèles de capital bancaire se fondent sur ce type d'argument pour justifier la présence de fonds propres dans le passif de la banque. Toutefois, la formalisation de la préférence pour le risque dans le choix de la composition de l'actif bancaire n'est pas présente ici, ce sera le cas dans la section consacrée au canal de la prise de risque.
} 
Dans MM, à la suite d'un choc technologique négatif affectant tous les entrepreneurs, certains d'entre eux sont dans l'incapacité de rembourser leur emprunt bancaire. L'actif de la banque se réduit. Afin de convaincre les ménages de continuer à lui confier leur épargne, la banque doit augmenter le ratio capital bancaire / total de l'actif. Cela est rendu possible, dans MM, par une chute de l'offre de crédit (ce qui réduit davantage les actifs de la banque) et dans $\mathrm{GKr}$, par la vente des actifs risqués (ce qui réduit davantage le prix des actifs risqués, et donc l'actif de la banque).

De plus, l'amplification du choc macroéconomique, à l'instar de BGG, passe par l'investissement: le crédit étant destiné à financer l'investissement des entrepreneurs, le « credit crunch » dans MM ou la chute du prix des actifs financiers dans GKr réduit l'achat de nouvelles machines, ce qui vient peser sur la croissance de la production dans les périodes suivantes. MM montrent que la présence du canal du capital bancaire amplifie l'effet des chocs technologiques sur la production, cette amplification étant significative par rapport à un simple modèle du type BGG (voir également Levieuge, 2009).

Cette propriété d'amplification est également présente dans le cas des chocs monétaires (MM ; Levieuge, 2009). Ces derniers affectent, via le taux d'intérêt certain, la rémunération des dépôts des ménages auprès de la banque, donc le coût de financement des banques. Le canal du capital bancaire amplifie les chocs agrégés lorsque le capital bancaire répond à une logique économique (GKr; MM; Levieuge, 2009) ou réglementaire (Van den Heuvel, 2002).

Ces résultats sont cohérents avec les études empiriques qui mettent en évidence l'impact de la situation bilancielle sur l'offre de crédit. De plus, le modèle de MM parvient à reproduire la contra-cyclicité du ratio de capital / prêt observée sur données américaines entre 1990 et 2005. La crise de 2007 est résumée dans leur modèle par un choc négatif sur le capital des banques. Toutefois, MM ne donnent pas de contenu empirique à un tel choc. Ils considèrent l'impact d'une baisse de $5 \%$ de la valeur du capital bancaire, ce qui peut correspondre, selon eux, à un épisode de tension financière.

Le choc sur le capital bancaire fait écho à l'idée en développement dans la littérature MEGIS selon laquelle le secteur financier ne serait pas seulement une source d'amplification de chocs agrégés mais également une source de chocs. Toutefois, aucun article ne tente de fournir avec rigueur une estimation empirique des chocs financiers susceptibles d'avoir déclenché la crise. L'estimation structurelle des modèles présentés dans la section 3 pourrait nous éclairer sur le contenu empirique des chocs financiers évoqués dans les MEGIS de cet article.

\section{b) la liquidité sur le marché interbancaire Gertler et Kiyotaki (2010) : canal du crédit interbancaire}

Le canal large du crédit place la richesse nette des emprunteurs non financiers au cœur de l'accélérateur financier. Le canal du crédit bancaire étend ce mécanisme aux intermédiaires financiers. Cette idée a été développée par Bernanke et Blinder (1988), selon lesquels, le mécanisme du canal du crédit bancaire repose sur l'obligation de réserves auprès de la banque centrale, réserves assises sur le montant des dépôts. Le resserrement de la contrainte de réserves obligatoires conduirait les banques commerciales à réduire le volume de leurs dépôts et donc le montant des crédits distribués. La crise actuelle invite à un réexamen du canal du crédit bancaire dans un contexte dans lequel les réserves auprès de la banque centrale ne font plus l'objet d'une obligation légale aux Etats-Unis, et restent faibles (2\%) en zone Euro. De plus, les intermédiaires financiers trouvent leurs financements non seulement auprès de la banque centrale mais également auprès d'autres banques. C'est le cas dans l'article de GK (2010). 
Dans leur modèle, les entrepreneurs se financent auprès des banques pour mener des projets d'investissement. L'observation de leur rendement ne pose aucune difficulté, contrairement au modèle de BGG. L'apparition de spread de taux prend donc sa source dans d'autres frictions financières. GK se fondent sur l'évolution exceptionnelle des spreads de taux sur le marché interbancaire pour justifier leur accent sur les difficultés de financement des banques dans leur modèle.

GK font l'hypothèse que la collecte de fonds par la banque commerciale est séquentielle.

- Tout d'abord, elles collectent des fonds auprès des ménages (marché du détail). GK reprennent l'idée, développée dans la section a), selon laquelle la banque peut décider de faire faillite et ne pas rembourser les dépôts des ménages.

- Ensuite, les banques sont soumises à des chocs de liquidité. Ces derniers créent des besoins ou des surplus de financement que les banques ne peuvent combler que via le marché interbancaire (marché de gros). Il leur est impossible de revenir vers les ménages pour augmenter leurs ressources car le marché du détail est fermé. Cet accès séquentiel aux ressources bancaires constitue une rigidité financière qui rappelle celle retenue par les modèles de participation limitée (Allen et Gale, 1994, 2007).

Bilan de l'intermédiaire financier dans GK (2010)

\begin{tabular}{|c|c|}
\hline Actif & Passif \\
\hline $\begin{array}{c}\text { Actions émises par les entrepreneurs } \\
\left(\text { rendement } \mathrm{R}^{\mathrm{k}}\right)\end{array}$ & $\begin{array}{c}\text { - Dépôts des ménages (rémunération au } \\
\text { taux certain R) } \\
\\
\text { - Dette interbancaire (rémunération au } \\
\text { taux certain } \mathrm{R}^{\mathrm{i}} \text { ) } \\
\text { - Fonds propres }\end{array}$ \\
\hline
\end{tabular}

Notons que le modèle de GK permet de rendre compte de l'évolution de trois taux d'intérêt : le taux créditeur (le taux d'intérêt certain $\mathrm{R}$ ), le taux débiteur $\mathrm{R}^{\mathrm{k}}$ et le taux interbancaire $\mathrm{R}^{\mathrm{i}}$. Un quatrième taux d'intérêt concerne le taux pratiqué par la banque centrale dans le cadre de sa politique de prêt à l'économie.

La dispersion des chocs de liquidité est présentée sous la forme d'îles. Les banques sont distribuées aléatoirement sur des îles sur lesquelles elles rencontrent des investisseurs. On retient l'hypothèse forte que les entreprises locales ne s'adressent qu'aux banques locales. Certaines îles reçoivent de manière aléatoire de nouvelles opportunités d'investissement qui accroissent les besoins de financement auprès des banques. Au moment où les opportunités d'investissement (et donc les besoins en financement) sont connues, les banques ont achevé de collecter les dépôts auprès des ménages. Les entreprises situées sur les îles qui présentent de nouvelles opportunités d'investissement sont en besoin de financement, c'est le cas également des banques locales. Ces dernières vont donc s'adresser aux banques des autres îles afin de se procurer le financement nécessaire. La distribution aléatoire des nouvelles opportunités d'investissement constitue une manière simple de modéliser le risque de liquidité subi par les banques.

Les emprunts interbancaires sont sujets également à un aléa moral, aléa qui vient se superposer à celui affectant les dépôts des épargnants. Les banques ont la possibilité de ne pas honorer une fraction exogène $\omega$ de leur dette interbancaire. Si c'est le cas, elles se déclarent 
en faillite après avoir reçu la rémunération de leurs actifs sur les projets d'investissement. Elles conservent donc la fraction $\theta$ de l'actif, net des dettes interbancaires non remboursées.

- En l'absence d'aléa moral sur la dette interbancaire, le marché interbancaire agit comme une assurance qui rend les banques identiques face au risque de liquidité. Les surplus de financement des unes viennent combler les besoins de financement des autres. Toutes les banques font face au même spread de taux $\left(\mathrm{R}^{\mathrm{k}}-\mathrm{R}\right)$, quel que soit leur risque de liquidité (leur localisation sur les îles). Le volume du crédit distribué dans l'économie est donc limité par la capacité d'endettement du secteur bancaire dans son ensemble.

- En présence d'aléa moral sur la dette interbancaire, les banques peuvent se déclarer en défaut de paiement sur leur dette interbancaire. Après avoir reçu le rendement du capital, elles font faillite et quittent alors le secteur financier.

o Sur les îles disposant des nouvelles opportunités d'investissement, les banques bénéficient de rendements élevés sur ses actifs (crédits octroyés aux entrepreneurs). La banque peut être tentée de renier ses dettes sauf si la poursuite de l'activité bancaire s'avère bien plus lucrative que la faillite, soit : ${ }^{21}$

$\begin{gathered}\text { Profit de la banque sans faillite } \\ \text { (la banque reçoit le rendement de ses actifs et } \\ \text { honore ses dettes) }\end{gathered}$
$\begin{aligned} & \text { élevé si } \\ & \text { - Coût de la dette faible (taux } \\ & \text { d'intérêt sur les dépôts des } \\ & \text { ménages et sur la dette }\end{aligned}$
$\begin{aligned} & \text { interbancaire) } \\ & \text { Rendement élevé sur actifs } \\ & \text { (la banque reçoit le rendement de ses actifs et } \\ & (1-\omega) \text { de la dette interbancaire et } \theta \text { de l'actif net) }\end{aligned}$
$\begin{aligned} & \text { de la banque } \\ & \text { Fonds propres élevés }\end{aligned}$
-

Cette inégalité est vérifiée, en particulier, si le profit de la banque en cas de faillite est faible, soit

- Le taux d'intérêt sur sa dette auprès des ménages (et donc la rémunération des dépôts) est bien moins élevé que le rendement sur ses actifs (les crédits octroyés aux entreprises). Cela se traduit par un spread de taux $\left(\mathrm{R}^{\mathrm{k}}-\mathrm{R}\right)$ élevé.

- Un spread de taux $\left(\mathrm{R}^{\mathrm{k}}-\mathrm{R}^{\mathrm{i}}\right)$ élevé, par un même raisonnement sur la dette interbancaire

- La banque doit réduire le montant des dépôts et de la dette interbancaire afin de respecter la contrainte de non faillite. Cela limite son profit en cas de faillite. Cette chute des ressources de la banque réduit l'offre de prêts, ce qui pèse sur le financement des projets d'investissement.

Sur les îles qui bénéficient de nouvelles opportunités d'investissement, les banques sont dites « contraintes » sur la liquidité.

o Sur les îles exploitant les investissements existants, les banques qui financent les investissements existants bénéficient d'un rendement moindre sur les actions des

\footnotetext{
${ }^{21}$ Cette présentation simplifiée néglige les aspects intertemporels dans la décision de la banque, liés en particulier à la séquence des marchés de financement (détail versus gros). Voir l'annexe C.
} 
entreprises. Le spread de taux $\left(\mathrm{R}^{\mathrm{k}}-\mathrm{R}\right)$ qui leur est appliqué est moindre que celui qui prévaut sur les îles disposant des nouvelles opportunités d'investissement.

Le modèle de GK introduit donc une hétérogénéité des banques sur le marché interbancaire, laquelle donne lieu à une dispersion des spread, avec en particulier, des spread élevés et persistants pour les banques en manque de liquidité, ces banques étant celles qui sont les plus susceptibles de se déclarer en défaut de paiement.

GK examinent les effets macroéconomiques d'un choc négatif sur la qualité du capital. En l'absence de secteur financier, le choc provoque une récession modérée et brève. La présence de frictions dans le secteur financier rend la récession plus marquée et plus longue, la crise financière affectant en premier lieu les banques contraintes sur la liquidité. Ces dernières subissent un coût de financement plus élevé. Dans un modèle multi-période, une propagation dynamique tend à accentuer l'effet initial de la baisse de la richesse nette de la banque. La baisse des dépôts réduit davantage la richesse nette de la banque, ce qui vient amplifier la chute du crédit bancaire et donc l'investissement. La sortie de crise passe par la reconstitution de la richesse nette de la banque, processus qui prend du temps. Cela vient prolonger la durée de la crise économique. GK montrent que le modèle prédit une récession durable lorsque l'étalonnage reflète de fortes frictions financières.

Le cadre théorique permet une évaluation quantitative de chocs financiers, tels que des chocs sur la richesse nette de la banque ou sur la fraction des actifs que la banque peut s'approprier en cas de faillite ( $\omega$ et $\theta$ ). Toutefois, GK ne procèdent pas à ce type d'exercice, sans doute faute de données précises sur l'ampleur de ces chocs dans les données. A ce jour, l'ensemble des implications quantitatives de ce modèle n'a pas encore été exploré.

Le modèle de GK propose une version moderne du canal du crédit bancaire, ce qui se traduit par une analyse fine des taux d'intérêt et de leurs spreads.

- Toutefois, on s'interroge sur la pertinence empirique de cette parabole des îles qui suppose en particulier la capacité d'identifier les îles, c'est-à-dire, les marchés soumis à des tensions. Or, Gilchrist et Zakrajek (2011) démontrent que le spread de taux ne saurait constituer un indicateur fiable de telles tensions. Ce point est essentiel dans le ciblage de l'intervention de la banque centrale.

- La crainte de la faillite bancaire conduit les mécanismes d'ajustement. Toutefois, la faillite des intermédiaires financiers est inexistante à l'équilibre ${ }^{22}$.

- Par ailleurs, par souci de simplicité, GK font également abstraction de la faillite des entreprises, point pourtant central dans le modèle fondateur de BGG.

- Il nous semble que l'efficacité des politiques monétaires non conventionnelles, menée dans l'article, est surestimée. En effet, les banques sont financées uniquement par une dette à court terme (les dépôts des ménages ou la dette interbancaire). Toute intervention de la banque centrale pour desserrer cette contrainte au passif via une baisse du taux de refinancement ou une acquisition d'actions dans le secteur bancaire a un fort impact sur l'activité des intermédiaires financiers. GK retiennent l'hypothèse peu réaliste d'une absence de la possibilité pour la banque de se financer par actions, de sorte que la banque est privée de la possibilité de répercuter ses pertes éventuellement enregistrées à l'actif par une moindre distribution de dividendes au passif. La présence d'actions au passif de la

\footnotetext{
${ }^{22} \mathrm{GK}$ font l'hypothèse que certains banquiers quittent l'activité de manière exogène. Toutefois, cette astuce inspirée de Gertler et Karadi (2011) est destinée à préserver le cadre d'un agent représentatif.
} 
banque vient limiter l'efficacité de la politique monétaire non conventionnelle. Ce point est développé par Gertler, Kiyotaki et Queralto (2010) présenté dans la section suivante.

Le modèle de GK ne fournit donc pas entièrement les clés pour analyser le risque systémique et ses liens avec la politique monétaire, éléments qui sont mieux pris en compte dans les contributions présentées dans la section suivante.

\section{2. 3. Le canal de la prise de risque}

La titrisation et le développement de la banque parallèle («shadow banking ») a profondément modifié le secteur bancaire dans la dernière décennie. Adrian et Shin (2010) soulignent que, la nature de l'activité des banques parallèles les incite à gérer leur bilan de façon à accroître leur exposition au risque lorsque le prix des actifs financiers augmente. Cet appétit accru pour le risque (" yield appetite ») constitue l'un des éléments essentiels du canal de la prise de risque. Ce dernier est d'abord évoqué par Rajan (2005) avant d'être développé par des économistes de la Banque des Règlements Internationaux (Borio et Zhu, 2008).

Dès 2005, Rajan considérait que la titrisation et le développement du "shadow banking " constituaient des évolutions majeures du secteur financier, évolutions susceptibles d'engendrer une prise de risque excessive. Le niveau très modéré des taux d'intérêt choisis par la Fed dans les années précédant la crise ont plafonné le rendement des actifs non risqués, ce qui a incité les acteurs du "shadow banking» à rechercher des rendements plus conséquents, avec une prise de risque significative. Un niveau de taux d'intérêt très modéré a également favorisé le financement des stratégies d'investissement par un endettement de court terme. L'effet de levier est alors au cœur de la stratégie de prise de risque.

Les éléments du canal de la prise de risque sont repris par Borio et Zhu (2008). Ces derniers mettent l'accent sur les mécanismes par lesquels une politique monétaire caractérisée par un taux d'intérêt faible peut inciter les banques à prendre des risques excessifs, comme si les périodes de stabilité économique diminuait l'aversion au risque. Le rapport du CAE (2011) rappelle les effets à l'œuvre dans le canal de la prise du risque. La définition de Borio et Zhu (2008) suggère une modification des préférences en fonction de la santé de l'économie, avec une sous-estimation du risque ou un optimisme excessif en période d'expansion. Borio et Zhu (2008), pas plus que Rajan (2005), ne proposent une modélisation de ces phénomènes. La formalisation de ces mécanismes, en vue d'une évaluation quantitative de leur impact sur la dynamique macroéconomique, est proposée dans les deux contributions que nous avons retenues. ${ }^{23}$ Ces travaux centrent leur analyse sur la transformation bancaire comme source du risque de faillite de la banque. L'intermédiaire financier optimise son bilan avec un passif composé d'une dette assortie d'un taux d'intérêt certain et un actif investi dans des actifs risqués.

\section{a) Gertler, Kiyotaki et Queralto (2010)}

L'article de GKQ étend GK et GKr et constitue la contribution la plus appropriée pour évaluer l'impact des politiques monétaires non conventionnelles. Cela tient à la richesse de la structure bilancielle considéré dans ce modèle.

\footnotetext{
${ }^{23}$ Adrian et Shin (2010) ainsi que Dubecq, Mojon et Ragot (2010) proposent des modélisations du canal de la prise de risque, toutefois dans un cadre à 2 périodes, sans quantification de l'interaction entre la prise de risque des banques et l'activité économique.
} 
GKQ analysent les décisions de la banque en matière de composition de son passif, ce dernier étant composé de 3 éléments :

- fonds propres (profit bancaire instantané réinvesti totalement dans la banque),

- actions émises par la banque

- dette de court terme (dépôts des ménages).

Le choix de la banque sur la taille et la composition de son bilan affecte son exposition au risque.

Bilan de l'intermédiaire financier dans GKQ (2010)

\begin{tabular}{|l|l|}
\hline Actif & Passif \\
\hline $\begin{array}{l}\text { - Actions émises par les agents non } \\
\text { financiers }\end{array}$ & - Dépôts des ménages \\
& $\begin{array}{c}\text { - Actions émises par l'intermédiaire } \\
\text { financier (« outside equity ») }\end{array}$ \\
& - Fonds propres (« inside equity ») \\
\hline
\end{tabular}

a1) Qu'est-ce qu'une banque qui manifeste une préférence pour le risque ? Une telle banque n'émettra que peu d'actions et s'endettera davantage (ratio dette / total actif élevé ou ratio actions / total actif faible). En effet, en cas de choc agrégé défavorable, la banque peut enregistrer des pertes significatives sur son actif (composé uniquement d'actions émises par les entrepreneurs qui financent ainsi des projets d'investissement) mais reste liée par une dette de court terme qu'elle doit payer au taux d'intérêt certain. La transformation bancaire opérée par la banque n'est plus possible et elle se déclare en faillite. Le canal de la prise de risque concerne donc les choix de la banque sur la composition de son passif uniquement. ${ }^{24}$

a2) Comment la banque choisit-elle la composition de son bilan ? Le choix de la banque se décompose de la manière suivante

- choix entre fonds propres (« inside equity ») versus fonds externes (actions aussi appelées « outside equity » et dette de court terme) : la proportion de fonds propres par rapport aux fonds externes repose sur l'aléa moral entre la banque et ses créanciers extérieurs (possibilité de la banque de s'approprier une fraction des actifs en cas de faillite). Dans GKQ, le banque choisit $\varphi$ le ratio fonds propres / total actifs.

- parmi les fonds externes, choix entre actions et dette de court terme. Une banque qui souhaite émettre davantage d'actions ( $x$, le ratio actions / total actifs, augmente)

o bénéficiera d'un financement plus souple : la banque peut ajuster la rémunération des actionnaires en fonction du rendement qu'elle obtient sur ses actifs. En revanche, la dette est assortie d'un taux d'intérêt sans risque.

o mais se trouve confrontée à une contrainte de participation (contrainte de non faillite de la banque) plus difficile à respecter : une plus forte proportion d'actions dans le passif de la banque (correspondant à une hausse de $x$ ) accroît, par hypothèse, $\Theta(x)$, la fraction des actifs que la banque peut s'approprier en cas de faillite, donc, le profit en cas de faillite. $\Theta(x)$ est supposé croissante en $x$. Cette hypothèse est justifiée par l'intuition selon laquelle il est plus difficile pour les actionnaires de déterminer le juste niveau des dividendes car ces derniers sont liés

\footnotetext{
${ }^{24}$ Dubecq, Mojon et Ragot (2010) analysent la prise de risque dans l'actif de la banque, cette dernière arbitrant entre titre risqué et titre non risqué (alors que, dans les deux contributions présentées dans cette section, la banque choisit la composition de son passif).
} 
aux profits bancaires, difficiles à évaluer pour les actionnaires. La forte proportion des actions dans son financement incite la banque à tricher sur ses dividendes et accroît son gain en cas de faillite. ${ }^{25}$

Comme dans $\mathrm{GKr}$ et GK, à l'équilibre, la faillite bancaire est exclue. La rémunération de la dette bancaire reste donc déterminée par un taux d'intérêt sans risque.

La banque centrale ne peut pas se déclarer en faillite. Toutefois, sa moindre expertise dans la connaissance des projets d'investissement des agents non financiers la rend moins efficace dans cette distribution de crédit directe à l'économie non financière. Ces coûts croissent en fonction du volume de prêts accordés par la banque centrale aux agents non financiers. Cela rappelle l'hypothèse retenue dans $\mathrm{CW}$.

a3) équilibre de long terme. Le choix de la composition bilancielle est en réalité intertemporel : parce que la banque prend cette décision avant la réalisation du choc agrégé, elle doit former des anticipations sur la politique monétaire et sa perception de l'incertitude dans l'économie. Le risque est au cœur du modèle (et donne lieu à une complexité computationnelle résumée dans l'annexe C) puisque le degré d'incertitude (résumé par l'écart-type des chocs agrégés) affecte le montant de $\varphi$ et $x$. GK illustrent ce point en comparant l'équilibre de long terme de 2 économies qui se distinguent par leur degré d'incertitude et montrent que,

- $x_{\text {faible incertitude }}<x_{\text {forte incertitude }}$ : dans une économie caractérisée par une forte incertitude, la banque a intérêt a émettre davantage d'actions de façon à se financer à un coût qui covarie avec le rendement sur l'actif bancaire. En période de récession, la banque verra le rendement de son actif chuter, chute qu'elle répercute sur son passif en réduisant le versement de dividendes

- $\varphi_{\text {faible incertitude }}>\varphi_{\text {forte incertitude }}$ : dans une économie caractérisée par une forte incertitude, la banque dispose de davantage de fonds propres de façon à convaincre les pourvoyeurs de fonds externes d'acheter ses titres (actions et titres de dette) malgré la possibilité de faillite bancaire.

Ces résultats suggèrent une plus grande fragilité bancaire en période de faible incertitude, ce qui viendrait expliquer le paradoxe de la tranquillité de Minsky selon lequel les prises de risque excessives s'accumulent au moment où l'économie semble aller le mieux. Dans GKQ, en période d'incertitude réduite, la plus grande prise de risque chez les banques les rend sensibles au choc agrégé.

a4) L'examen de la dynamique macroéconomique à la suite d'une chute inattendue et temporaire sur la qualité du capital fait apparaître deux amplifications

- i) La première est similaire à celle de GK et GKr. Une chute de la qualité du capital réduit le rendement sur l'actif de la banque et donc sa richesse nette (constituée des profits bancaires). Afin de convaincre les ménages de conserver leurs avoirs dans la banque (contrainte de non faillite), la banque doit réduire la taille de son actif, ce qui l'amène à les

\footnotetext{
${ }^{25}$ Dans GKr et GK, la banque choisit le montant de fonds propres de façon à convaincre le ménage de lui confier son épargne. La contrainte de participation formalise cette contrainte par laquelle le profit de la banque en cas de non faillite est supérieur à celui qui prévaut en cas de faillite. Cette dernière valeur dépend de l'ampleur des frictions financières, résumées par la fraction des actifs que la banque peut conserver en cas de faillite. GKQ (2010) étendent le modèle de GK en rendant cette fraction $\Theta(x)$ endogène. Ils la supposent croissante en fonction de $x$, la proportion d'actions dans le passif de la banque.
} 
vendre («fire sale») . Cela provoque une chute du prix de l'actif qui vient, à son tour, amplifier la baisse de la valeur de l'actif de la banque. L'ampleur de cet effet est réglée par le ratio d'endettement de la banque. Une banque plus endettée procèdera à une réduction plus marquée de son actif afin de respecter la contrainte de non faillite.

- ii) La seconde constitue l'innovation de cet article et tient à la présence d'actions dans le passif de la banque.

o Dans l'économie caractérisée par un risque modéré, la banque choisit d'une structure bilancielle fragile (une proportion élevée de dépôts dans son passif). Toute variation du rendement de son actif contraint la banque à une baisse significative de son actif afin de respecter la contrainte de non faillite. Ce scénario équivaut au cas envisagé par GK et GKr qui excluent le financement bancaire par actions.

o Dans l'économie caractérisée par un risque élevé, la présence d'une forte proportion d'actions dans le passif de la banque agit comme un coussin. Grâce à la réduction des actions dans le passif de la banque, cette dernière parvient à réduire $x$, ce qui réduit, par hypothèse, la fraction $\Theta(x)$ des actifs que la banque peut s'approprier en cas de faillite. En baissant $x$, l'intermédiaire financier respecte la contrainte de non faillite.

Si les mécanismes économiques sont clairs, leur adéquation quantitative aux données reste à affiner. Il reste à mener le travail de mise en correspondance des chocs réels et financiers observés dans les données au regard des effets macroéconomiques observés et prédits par le modèle.

\section{b) La fragilité bancaire : Angeloni et Faia (2010)}

Angeloni et Faia (2010) développent un cadre dans lequel les banques peuvent faire faillite. Ce point constitue l'originalité de ce travail par rapport aux autres travaux présentés dans cette revue de la littérature. Le modèle d'AF intègre les mécanismes de Diamond et Rajan (DR) (2000) dans un MEGIS qui, à l'équilibre, inclut des banques fragiles car exposées au risque de faillite.

Dans DR, la banque a pour rôle de fournir des fonds à des emprunteurs " difficiles » et des liquidités à des ménages qui lui confient leurs avoirs. La banque doit développer des qualifications spécifiques pour être capable d'inciter l'emprunteur à rembourser (la banque est " relationship lender »). Du côté de ses ressources, la banque pourrait également extraire une rente sur les dépôts mais la possibilité de « runs » le lui interdit. Cette menace de "runs » contraint la banque dans la collecte de ses ressources. Les fonctions de la banque sont complémentaires. Sans les qualifications spécifiques de la banque, l'entrepreneur est dans l'incapacité de trouver des financements, et, sans la possibilité de runs, la banque serait dans l'impossibilité de financer ses activités de distribution de crédit. La banque a un rôle de fournisseur de liquidités (transformation bancaire) : elle est capable de servir les ménages qui disposent ainsi d'un actif liquide à partir de crédits distribués, crédits par nature illiquides.

- En l'absence d'incertitude sur le rendement des projets financés par la distribution de crédits, la structure optimale du bilan de la banque implique une composition du passif à 100\% de dépôts bancaires. La banque maximise son actif en transférant aux détenteurs des dépôts bancaires les gains sur les crédits.

- En revanche, en présence d'incertitude, la banque ignore si elle sera capable de recueillir assez de ressources sur ses actifs pour servir ses créanciers - déposants. Le sachant, ces derniers peuvent céder à la panique : la banque est soumise au risque de « run ». Afin de convaincre les ménages de conserver les dépôts dans la banque, cette dernière a intérêt à disposer d'un capital propre. Ce dernier constitue effectivement une garantie pour les 
ménages déposants de récupérer leurs avoirs car les actionnaires de la banque sont servis en dernier en cas de faillite de la banque. La présence d'actionnaires ayant des droits distincts des déposants implique une modélisation des choix de la banque par un gérant, distinct des créanciers de l'intermédiaire financier (ménages et actionnaires).

A l'instar des modèles qui incluent le canal du capital bancaire, la banque détermine un montant de capital optimal pour convaincre les déposants de lui confier leurs avoirs. La différence entre le canal du capital bancaire et la fragilité bancaire développée dans cette section réside dans la liquidité.

- Dans les extensions du modèle BGG, les dépôts bancaires sont des stocks prédéterminés. Leur liquidité reste donc limitée. qu'il s'agisse de $\mathrm{KM}, \mathrm{BGG}, \mathrm{CW}, \mathrm{GKr}$ ou GK, les dépôts des ménages choisis en début de période constituent des ressources pour la banque jusqu'à la période suivante, date à laquelle le choix des dépôts est effectué à nouveau. Dans les modèles précédents, le choix de la séquence des décisions des agents exclut la possibilité de bank run. La possibilité de faillite bancaire soumet la banque à une discipline, laquelle exclut, à l'équilibre, la faillite bancaire.

- En revanche, le principe central de DR réside dans la possibilité de faillite de la banque liée à des paniques bancaires. Cela suppose une extrême liquidité des dépôts, hypothèse plus réaliste et cohérente avec les événements de 2008 (cas de Northen Rock en GrandeBretagne).

En pratique, dans le modèle d'AF, le modèle se présente de la manière suivante. Le rendement des projets financés par la banque est incertain. Dès que les rendements des projets sont connus, s'ils sont insuffisants, les déposants peuvent retirer leurs avoirs bancaires, cela implique la faillite de la banque. Cette dernière doit liquider ses actifs pour satisfaire la demande de liquidité par ses déposants. En cas de faillite, la banque commerciale ne bénéficie pas d'un dispositif d'urgence de la part de la banque centrale. AF ne visent pas à étudier l'impact des politiques non conventionnelles de refinancement des intermédiaires financiers, même si leur modèle de risque systémique endogène s'y prête.

Bilan de l'intermédiaire financier dans AF (2010)

\begin{tabular}{|l|l|}
\hline Actif & \\
\hline $\begin{array}{c}\text { - Actions émises par les agents non } \\
\text { financiers }\end{array}$ & - Dépôts des ménages \\
& - Fonds propres \\
\hline
\end{tabular}

Dans AF, le gérant choisit la structure optimale du bilan de façon à maximiser les gains espérés des déposants et des actionnaires. Le ratio dépôt / prêts est une fonction

- décroissante du taux d'intérêt payé par la banque sur les dépôts : une hausse du taux d'intérêt accroît la probabilité de run, ce qui incite le gérant de la banque à réduire le ratio de dépôts.

- croissante du rendement attendu sur les actifs de la banque: une hausse de ce rendement se traduit par des profits bancaires plus importants en l'absence de run, ce qui incite le gérant à accroître sa collecte de dépôts.

- croissante du pouvoir de négociation des déposants : lorsque la part que les déposants obtiennent en cas de liquidation des actifs de la banque augmente, le gérant peut collecter davantage de dépôts 
Le risque de faillite, sachant les décisions d'optimisation de la banque, est positif à l'équilibre, et ce, quel que soit l'étalonnage retenu. Cela signifie que, compte tenu de la rentabilité de son activité, malgré le risque de faillite, la banque choisira toujours un montant de dépôts bancaires supérieur à celui qui exclut le risque de faillite. L'intermédiation financière conduit intrinsèquement à une collecte de dépôts et une transformation bancaire comportant, de manière inévitable, un risque de faillite. L'article de $\mathrm{AF}$ le rappelle. Ce résultat à l'équilibre général est également cohérent avec l'argument de DR. En l'absence de risque de faillite, le run sur les dépôts est exclu. La banque a donc intérêt à ne pas disposer de fonds propres et à choisir une composition de passif uniquement composée de dépôts.

Puisque le risque de faillite bancaire n'est pas nul à l'équilibre, le taux d'intérêt rémunérant les dépôts devrait intégrer une prime de risque. Cela n'est pas le cas dans la version de AF et constitue sans doute une limite de leur modèle. ${ }^{26}$

Le modèle prédit la dynamique macroéconomique suivante :

- Suite à un choc positif de productivité, la hausse de la demande d'investissement accroît le $\mathrm{Q}$ de Tobin, ce qui réduit le rendement des actifs. Le fléchissement de l'inflation conduit la banque centrale à baisser le taux d'intérêt nominal (règle de Taylor), laquelle conduit la banque commerciale à collecter davantage de dépôts, ce qui accroît la probabilité de bank run. Ce cas illustre le canal de la prise de risque, dans lequel la détente monétaire opérée par la banque centrale incite l'intermédiaire financier à adopter une composition de bilan plus fragile (composée de davantage de dépôts).

- A la suite d'une contraction de la politique monétaire, l'investissement et le Q de Tobin baissent, ce qui accroît le rendement des actifs bancaires. Les banques choisissent de collecter moins de dépôts. Le bilan des banques est réorienté vers une composition moins risquée. Le canal du risque joue ainsi dans le sens inverse : la banque adopte une structure de bilan moins risquée à la suite de la politique monétaire restrictive.

Au terme de cette section, nous présentons un tableau résumant l'ensemble des contributions (tableau 1) et évaluons (dans le tableau 2) la capacité des développements récents à relever les défis énoncés dans la section 3.1.

Tableau 1 : Résumé des contributions

(voir à la fin de l'article)

Tableau 2 : contributions de la littérature au regard des défis suscités par la crise

\begin{tabular}{|c|l|}
\hline a) défi quantitatif & Articles \\
\hline - chocs financiers & $\begin{array}{l}\text { Meh et Moran (2010); Christiano, Motto et Rostagno (2010); } \\
\text { Curdia et Woodford (2010); Angeloni et Faia (2010) } \\
\text { - credit crunch / firesale }\end{array}$ \\
- spread de taux persistants & $\begin{array}{l}\text { Curdia et Woodford (2009); Gertler et Kiyotaki (2010); Gertler } \\
\text { et Karadi (2011); Gertler, Kiyotaki et Queralto (2010) }\end{array}$
\end{tabular}

\footnotetext{
${ }^{26}$ Nous remercions un rapporteur pour cette remarque. Si le ménage place ses avoirs dans plusieurs banques, il peut parvenir à parfaitement diversifier le risque de faillite bancaire de sorte que la rémunération sur ses dépôts bancaires est le taux d'intérêt certain. Cette hypothèse, qui n'est pas présentée dans AF, rappelle celle retenue dans BGG par laquelle la banque qui subit un risque de défaut de paiement sur les crédits octroyés est capable de rémunérer les dépôts des ménages au taux d'intérêt certain grâce à une parfaite diversification du risque.
} 


\begin{tabular}{|c|c|}
\hline - dynamique non linéaire & - \\
\hline $\begin{array}{l}\text { b) Evolution du secteur financier } \\
\text { - } \text { Titrisation } \\
\text { - Information floue sur le risque } \\
\text { - Shadow banking }\end{array}$ & $\begin{array}{l}-{ }^{a)} \\
-b) \\
\text { - c) }\end{array}$ \\
\hline $\begin{array}{l}\text { c) politiques économiques } \\
\text { - Politiques non conventionnelles } \\
0 \quad \text { Quantitative easing } \\
0 \quad \text { Credit Easing } \\
\text { - Exit strategy } \\
\text { - Stabilité financière }\end{array}$ & $\begin{array}{l}\text { Curdia et Woodford (2010); Gertler et Karadi (2011) } \\
\text { Gertler et Karadi (2011); Gertler et Kiyotaki (2010); Gertler, } \\
\text { Kiyotaki et Queralto (2010) } \\
\text { Angeloni, Faia et Winkler (2010) } \\
\text { Angeloni et Faia (2010); Curdia et Woodford (2010) }\end{array}$ \\
\hline
\end{tabular}

a) Voir Faia (2011) et Chari, Shourideh, Zettlin-Jones (2010) non présentés dans cet article

b) Voir Dubecq, Mojon et Ragot (2010) pour une $1^{\text {ère }}$ modélisation, sans analyse de l'impact macroéconomique de la prise de risque

c) A ce jour, non disponible mais un modèle à la GK(2010), GKr(2011), GKQ(2010) sans dépôts des ménages pourrait être considéré comme une modélisation de shadow banking

\section{Implications en termes de politique économique}

Les années précédant la crise ont été marquées par la « divine coïncidence » (Blanchard et Gali, 2007) selon laquelle la politique monétaire, en se concentrant que la stabilité du taux d'inflation, contribue une stabilité macroéconomique et financière. La crise financière remet en cause une telle stratégie. La stabilité de l'inflation et de l'ensemble des variables macroéconomiques n'ont pas empêché (voire, pour les partisans du canal de la prise de risque, ont porté les germes de) la crise. La stabilité financière et la stabilité de l'inflation constituent deux objectifs distincts. Se pose alors la question de savoir si ces 2 objectifs relèvent de la compétence de la banque centrale seule ou de la banque centrale et d'un organisme indépendant.

La séparation des rôles est-elle préférable à la centralisation ? Nous ne reviendrons pas sur les éléments de ce débat, synthétisés dans le rapport du CAE (2011). Il conviendrait de comparer les propriétés d'une économie dans laquelle le principe de séparation est appliqué à une autre dans laquelle ce n'est pas le cas. Nous n'avons pas connaissance de tels travaux dans la littérature MEGIS. Il s'agit certainement d'un champ à explorer.

Dans cette section, nous considérons donc que le choix sur la pertinence du principe de séparation a été effectué. Nous nous interrogerons sur les recommandations de politique économique lorsque la stabilité financière et la stabilité de l'inflation relèvent de la banque centrale seule (section 4. 1) en distinguant (a) la prévention des crises financières de (b) la gestion de la crise elle-même par les politiques non conventionnelles. Nous envisagerons ensuite le cas de la gestion des 2 objectifs par 2 organismes distincts (section 4. 2.). 


\section{1. Politique monétaire}

\section{1. 1. La prévention de la crise financière}

Une telle prévention se traduit par l'usage d'une règle de Taylor augmentée d'indicateurs de «stress financier ». Ce débat sur l'élargissement de la règle de Taylor a été évoqué dans la littérature au début des années 2000 au sujet du prix des actifs financiers ou immobiliers ${ }^{27}$. Ce débat se heurte à une difficulté majeure, celle de la détection d'un emballement du prix des actifs. Cette pierre d'achoppement est également présente dans la politique de prévention de la crise financière. Si la règle de Taylor est augmentée d'indicateurs de "stress financiers ", quels qu'ils soient, comment distinguer une croissance du crédit excessive d'une expansion simplement conduite par une embellie économique?

Une règle de Taylor augmentée tiendrait compte des éléments suivants :

- le taux de croissance du crédit (« credit adjusted Taylor rule »): distinct de son rôle dans la prévision du taux d'inflation. Christiano, Ilut, Motto et Rostagno (2010) remarquent que, sur données américaines et japonaises, les périodes d'expansion boursière sont caractérisées par une inflation modérée et un taux de croissance des crédits élevés. Une règle de Taylor centrée uniquement sur la stabilité de l'inflation pourrait donc encourager le boom boursier. En revanche, une règle de Taylor augmentée du taux de croissance du crédit pourrait contribuer à réduire la volatilité de la dynamique macroéconomique et du prix des actifs financiers. Cette intuition est vérifiée dans leur modèle, inspiré de Christiano, Motto, Rostagno (2010).

- Les variations des spreads de taux («spread-adjusted Taylor rule »): En présence de financement intermédié, le taux directeur n'est plus le déterminant principal du coût de financement dans l'économie. En effet, les entrepreneurs qui se financent auprès des intermédiaires financiers paient une prime de financement externe, laquelle varie au cours du cycle économique. La prise ne compte de ce spread (différence entre taux débiteur et créditeur de la banque) permettrait de prendre en compte, dans la règle de Taylor, de l'impact de la politique monétaire sur la prime de financement externe. CW ne sont pas convaincus par cet argument. Leur évaluation montre que le gain en termes de stabilisation macroéconomique d'une règle de Taylor augmentée n'est pas quantitativement significatif et dépend de la nature des chocs dans l'économie. $\mathrm{CW}$ ne trouvent pas non plus de gain en termes de bien-être à l'inclusion du taux de croissance du crédit dans la règle de Taylor, plutôt que le spread de taux. Cela est lié à la relation directe (et ad hoc) dans leur modèle entre le spread et le volume de crédits. L'usage de l'un ou de l'autre dans la règle de Taylor a donc peu d'impact sur la dynamique macro économique. $\mathrm{CW}$ plaident donc en faveur d'un ciblage d'inflation et d'activité, seule stratégie susceptible de stabiliser l'économie quelle que soit la nature des chocs agrégés. La prise en compte des spreads de taux et de la croissance du crédit dans la détermination des cibles retenues permettra une stabilisation macroéconomique qui tient compte des frictions financières.

- AF considèrent des ratios d'endettement imposé selon trois régimes différentes : i) Un ratio d'endettement fixe (qui est une approximation du ratio de Bâle I) ; ii) un ratio contracyclique (comme Bâle II), un tel ratio tend à amplifier les cycles puisque

\footnotetext{
${ }^{27}$ Voir les contributions du colloque « Asset Prices and Monetary Policy », European Central Bank, 2003. Nous remercions un rapporteur pour cette référence.
} 
l'endettement de la banque et la distribution de crédit s'accentuent en expansion et iii) un régime hypothétique qui impose un ratio procyclique (Bâle III), un tel ratio vient modérer l'ampleur des cycles puisque l'endettement de la banque baisse en expansion. L'analyse vise à étudier la politique monétaire en présence des 3 régimes de réglementation bancaire. En particulier, AF ont pour objectif de mesurer l'effet sur le bien - être et la volatilité macroéconomique (activité et inflation) de différentes règles de Taylor. Il s'agit de déterminer l'intérêt d'inclure dans la fonction de réaction de la banque centrale le prix des actifs (le $\mathrm{Q}$ de Tobin) ou le ratio d'endettement des banques commerciales. AF montrent qu'une règle de Taylor tenant compte de la structure du bilan des banques serait capable de satisfaire les 3 critères retenus (maximiser le bien-être du consommateur et stabiliser l'activité et l'inflation). Les auteurs restent mesurés toutefois dans leurs conclusions car les prédictions quantitatives sont sensibles à l'étalonnage retenu. Les auteurs suggèrent que, au-delà de ses objectifs de stabilisation de l'inflation et de l'activité, la banque centrale, dans la conduite de sa politique monétaire contracyclique, pourrait prendre en compte les ratios d'endettement des banques commerciales.

Doit-on introduire dans la règle de Taylor des indicateurs de «stress financier »? La réponse à cette question passe par la recherche d'une stratégie de fixation des taux directeurs qui demeure stabilisante quelle que soit la nature des chocs agrégés et des frictions dans l'économie. La banque centrale se doit de définir une politique monétaire robuste à l'origine de l'incertitude et la modélisation de l'économie. Cette recherche est menée par exemple par Taylor et Wieland (2009) pour des modèles qui ne formalisent pas le secteur financier. Il conviendrait d'étendre leur démarche aux contributions récentes présentées dans cette revue de la littérature.

Or, d'après CW, une règle de Taylor robuste et augmentée d'indicateurs financiers n'existe pas. Pour étayer cette conclusion, une étude comparant les recommandations des modèles MEGIS post-BGG, intégrant le secteur bancaire, serait précieuse. Elle reste à ce jour à examiner.

\section{1. 2. La gestion de la crise}

Dans cette sous-section, nous présenterons les résultats des modèles MEGIS concernant 2 aspects de la gestion de la crise : l'évaluation de l'impact des politiques monétaires non conventionnelles et les recommandations en termes de stratégie de sortie de crise (« exit strategy »).

\section{a) politiques monétaires non conventionnelles}

a1) CW : « credit easing » préféré au « quantitative easing »

Dans $\mathrm{CW}$, le passif de la banque centrale se compose des réserves des intermédiaires financiers, tandis que son actif est composé de prêts directs au secteur privé et des titres émis par l'Etat rémunéré au taux $i_{t}^{d}$. L'usage des politiques non conventionnelles équivaut pour la banque centrale à modifier le montant des réserves (ce faisant, elle choisit la taille de son bilan, il s'agit donc du « quantitative easing ») ou celui du crédit aux agents privés (ce faisant, elle choisit la composition de son bilan, il s'agit donc du « credit easing »).

En ce qui concerne la politique optimale sur les réserves : la banque centrale doit ajuster le montant des réserves de façon à égaliser la rémunération des réserves au taux d'intérêt sur les 
dépôts bancaires $i_{t}^{d}=i_{t}^{m}$. L'intuition de ce résultat réside dans l'hypothèse de coûts opérationnels de l'intermédiaire financier croissants avec le montant des réserves. Ce coût donne lieu à un écart entre $i_{t}^{d}$ et $i_{t}^{m}$. Ce spread de taux mesure donc l'inefficacité liée à la présence des coûts opérationnels. En ciblant, $i_{t}^{d}=i_{t}^{m}$, la banque centrale vise à faire disparaître cette inefficacité. Elle ajuste donc le montant des réserves de façon à minimiser la perte de ressources liées aux coûts opérationnels.

Pour $\mathrm{CW}$, la définition du « quantitative easing » retenue dans leur article correspond bien à la politique d'accroissement de la base monétaire menée par le Japon en 2001-2006. Dans ce cas, le «quantitative easing» ne saurait constituer un instrument de politique monétaire pertinent car il doit être utilisé uniquement pour réduire la distorsion inhérente aux coûts opérationnels des banques.

Quant au "credit easing»: CW supposent une inefficacité relative de la banque centrale dans la distribution directe de crédit aux agents privés non financiers. Si l'étalonnage du modèle retient une forte inefficacité de la banque centrale par rapport aux banques commerciales, le «credit easing » est tout à fait exclu.

Toutefois, le prêt direct aux agents privés non financiers peut être envisagé, à titre exceptionnel, et uniquement en cas de chocs financiers majeurs. Par la hausse des prêts directs aux agents privés non financiers, l'activité économique peut être soutenue tout en évitant qu'elle ne soit pénalisée par la chute de l'offre de crédit de la banque commerciale. Cette dernière, grâce à la baisse de sa distribution de crédit, voit ses coûts de fonctionnement se réduire (via l'hypothèse de lien direct entre le volume de prêt et les coûts opérationnels), ce qui tend à restaurer graduellement les profits de la banque commerciale.

En l'absence de chocs financiers majeurs, l'usage de la composition du bilan de la banque centrale en tant qu'instrument de stabilisation macroéconomique ne saurait constituer une pratique courante. CW tendent à conforter la préconisation "Treasury only » selon laquelle l'actif de la banque centrale ne devrait être composé que de titres d'Etat, rendant toute décision concernant la composition de l'actif de la banque centrale non pertinente. ${ }^{28}$

a2) Efficacité relative des différents volets de «credit easing» et effet pervers d'une telle mesure

GK (2010) examinent 3 volets de la politique monétaire non conventionnelle, constitutifs du « credit easing ». Le premier a trait à la substitution de la banque centrale aux banques commerciales dans le financement des agents non financiers («direct lending »). Le $2^{\text {ème }}$ comprend le financement des banques commerciales («discount window lending »). Le dernier a trait à l'acquisition de capital bancaire par la banque centrale (« equity injections»). En particulier, GK (2010) soulignent la grande efficacité de la politique de direct lending par rapport aux 2 autres volets de la politique de credit easing. Toutefois, les remarques exposées à la fin de la section 3.2.2.b) (en particulier, possibilité, dans le modèle, d'identifier les marchés jugés illiquides et absence de financement des banques commerciales par émission d'actions) nous conduisent à conclure à une surestimation de l'efficacité de ce type de politique.

\footnotetext{
${ }^{28}$ Goodfriend (2009) rappelle l'intérêt du « Treasury only », même dans le cas de la crise financière de 2008.
} 
GKQ dévoilent un effet pervers du «credit easing »: Une politique de "credit easing » systématique affecte la perception du risque par les banques. La certitude que la banque centrale va se substituer aux banques pour financer l'économie incite les banques commerciales à adopter une structure de passif plus tournée vers la dette de court terme, donc plus risquée. Une politique macro-prudientielle appropriée doit inciter les banques à tenir compte de l'impact macroéconomique de leur prise de risque. GKQ proposent de délaisser le «credit easing » au profit d'une subvention à l'émission d'actions, dont le montant serait croissant avec le ratio x (actions / total actif) de chaque banque de façon à s'assurer que la banque dispose de suffisamment de dette contingente (actions) par rapport à sa dette non contingente (dont le coût est le taux d'intérêt). Les simulations quantitatives montrent que la politique macro-prudentielle tend à stabiliser l'économie en réduisant la fragilité bancaire ( $x$ augmente), et ce quel que soit le degré d'incertitude dans l'économie. La contribution de GKQ est particulièrement intéressante car elle met en lumière la réponse du secteur bancaire à la politique monétaire, et fait écho aux inquiétudes relatives à l'aléa moral suscité par les opérations de renflouement des banques et l'intervention massive des banques centrales via le « credit easing ».

\section{b) la stratégie de sortie}

La politique monétaire «non conventionnelle » est par nature transitoire car elle est mise en place lorsque les mesures conventionnelles de fixation des taux directeurs s'avèrent inefficaces. Se pose alors la question des stratégies de sortie. Une fin trop brutale ou prématurée de la politique monétaire non conventionnelle risque de prolonger la crise tandis qu'un soutien prolongé au secteur financier est susceptible d'encourager les intermédiaires financiers à reprendre une stratégie de recherche de rendement, et donc d'expansion excessive d'actifs, répétant ainsi le schéma de la crise financière. Les modèles MEGIS peuvent éclairer ce débat. Angeloni, Faia et Winkler (2010) proposent d'analyser les stratégies de sortie de crise dans un modèle de fragilité financière présenté supra. Ils incluent dans ce modèle une politique budgétaire de sorte que la sortie de crise nécessite une coordination entre politiques monétaire et budgétaire.

Leur article souligne la nécessité d'une stratégie de sortie. En effet, si la politique monétaire non conventionnelle se poursuit, la baisse permanente du taux d'intérêt certain qui en résulte incite les banques à collecter davantage de dépôts bancaires puisque leur rémunération baisse. La hausse du ratio dépôts / capital bancaire accroît le risque de run et donc le risque de faillite bancaire. Les conditions menant à la crise financière de 2007 sont re-créées.

$\mathrm{Au}$ terme de l'examen des différents scénarios envisagés (sortie graduelle versus brutale, annoncée versus surprise, sortie budgétaire avant la sortie monétaire et vice versa), les auteurs soulignent les caractéristiques d'une stratégie de sortie réussie : une politique rapide (mise en œuvre franche, concentrée dans une période courte; stratégie préférée à une mise en œuvre graduelle), une modifications durable des objectif de long terme (un engagement clair sur la stabilité financière pour la politique monétaire). La mise en œuvre brutale de la stratégie de sortie vise à inciter fortement les banques à ajuster rapidement leur passif vers une composition moins risquée.

\section{2. Coordination entre politique monétaire et politique macro- prudentielle}

Dans la section 4.1., nous avons présenté les recommandations des travaux qui prenaient pour acquis le principe de non séparation selon lequel la banque centrale assume le double rôle de stabilité financière et de stabilité de l'inflation. Or, comme le rappellent Galati et Moessner 
(2011), la stabilité financière est multi-dimensionnelle. Au-delà du consensus selon lequel la stabilité financière signifie une réduction de la probabilité d'une crise financière, les objectifs d'un organisme chargé de la stabilité financière incluraient

- la consolidation d'un système financier robuste aux chocs exceptionnels (« stress tests »)

- la continuité des services d'intermédiation (paiement, intermédiation de crédit, et assurance, banque d'Angleterre, 2009)

- la lutte contre les sous-estimations du risques inhérentes aux périodes d'expansion (Bruneimer et al. 2009) ou

- la lutte contre les bulles financières (Landau, 2009).

La politique macroprudentielle est menée à l'aide d'instruments spécifiques (Goodhart, 2011) fondés sur les ratios de fonds propres et les ratios de liquidité. Se pose également la question de la réglementation du secteur du « shadow banking » (Hanson, Kashyap et Stein, 2011).

Quels que soient les choix retenus en termes de réglementation, un durcissement des contraintes réglementaires contribuera à une restructuration ou à une re-définition des stratégies managériales des intermédiaires financiers. La réponse des acteurs du secteur financier aux modifications de réglementation doit être incluse dans une évaluation des politiques macro-prudentielles dans le cadre d'un modèle MEGIS. Nous avons évoqué ce point dans GKQ.

Deux autres contributions illustrent l'apport potentiel des MEGIS pour éclairer ces débats de politique économique.

La première est celle de Levieuge (2009) dans un modèle BGG avec capital bancaire (section 3.2.2. de cet article). L'auteur évalue l'impact de l'introduction du provisionnement dynamique (prévu par ailleurs par Bâle III) sur la dynamique macroéconomique. Par ce dispositif, la banque procède au provisionnement dès que le crédit est octroyé, de façon à anticiper dans son bilan la perte éventuelle inscrite dans son actif liée à la probabilité future de défaut de paiement. En revanche, en cas de provisionnement statique, un lien est établi entre le volume de provisions et de crédits octroyés à la même date. La banque n'est pas amenée à anticiper les pertes qu'elle prévoit éventuellement dans le futur.

Le provisionnement dynamique tendrait à réduire la procyclicité inhérente à l'activité d'intermédiation financière : en période d'expansion, l'octroi de crédit serait freiné par la constitution des provisions qui en découlent, provisions qui viendraient modérer l'impact sur le bilan de la banque des pertes sur les crédits enregistrées en période de récession. Le provisionnement dynamique modérerait les effets d'amplification inhérents au canal du capital bancaire. Le modèle de Levieuge (2009) confirme cette intuition avec une réduction substantielle de la volatilité des fluctuations du PIB et de l'inflation, quel que soit le choc considéré. La réduction de la volatilité est constatée par rapport au cas de provisionnement statique ou à l'absence de provisionnement. Le bénéfice de la stabilisation macroéconomique doit être pondéré par le coût associé, lié à une hausse du coût de financement externe pour les entrepreneurs. Le provisionnement dynamique est coûteux pour les banques, coût qu'elles répercutent sur le taux d'intérêt qu'elles appliquent aux crédits.

L'analyse de Levieuge (2009) appelle deux remarques. Tout d'abord, les banques espagnoles étaient soumises à l'obligation de provisionnement dynamique avant la crise, sans pour autant échapper à la crise financière. Une évaluation de la politique macro-prudentielle doit tenir compte de l'ensemble de ses instruments. De plus, le modèle de Levieuge (2009) exclut les 
faillites bancaires, donc, le risque systémique. Seul un modèle incluant la faillite bancaire à l'équilibre peut constituer un point de départ pertinent pour l'analyse de la stabilité financière.

La seconde contribution est celle d'Angeloni, Faia et Winkler (section 4.1.2. b) de cet article) qui s'interrogent sur l'impact macroéconomique de la modification des règles de capital réglementaire (Bâle III). L'évolution du ratio de Bâle est considérée comme donnée (elle n'est donc pas choisie dans le cadre de la politique macro-prudentielle). Un ratio de capital plus exigeant peut aggraver les difficultés des banques commerciales mais contribue également à restaurer la confiance des ménages dans le système financier, réduisant la probabilité de panique bancaire. AFW montrent que ce dernier effet domine. La difficulté de l'exercice réside dans la transposition aussi précise que possible des règles de Bâle dans le modèle, sans procéder à des simplifications excessives. L'analyse de AFW relève ce défi.

Au-delà de l'introduction des outils de la politique macro-prudentielle dans un modèle MEGIS, il reste à considérer la question de la coordination entre la banque centrale et un organisme indépendant tourné vers la stabilité financière. Un MEGIS analysant cette question devra s'interroger sur la séquence optimale de la prise de décision (le choix de la politique monétaire avant ou après le choix de la réglementation bancaire, le timing de ce jeu sera crucial dans les résultats). Les modèles MEGIS ne fournissent pas à ce jour d'éléments de réponse sur ces questions.

\section{Conclusion}

Les contributions présentées dans cet article explorent des pistes de recherche prometteuses dans la modélisation de l'intermédiaire financier et de l'interaction entre sphères réelle et financière. Le tableau 2 suggère que les travaux présentés dans cet article ont relevé les défis suscités par la crise. Les deux articles qui explorent le canal de la prise de risque constituent à notre sens les contributions les plus significatives car elles placent le risque au cœur de la compréhension de l'activité de l'intermédiaire financier. Elles proposent donc des outils privilégiés pour analyser la stabilité financière.

Ces avancées théoriques doivent cependant être mieux étayées empiriquement.

- Tout d'abord, l'évaluation quantitative des modèles proposés n'est pas encore assez précise quant à leur capacité à rendre compte

o des propriétés cycliques du comportement bancaire (en particulier sur leur ratio d'endettement, leur recours aux émissions d'actions, ..., variables qui sont au centre des nouvelles modélisations). Nous aurions aimé trouver une confrontation systématique des propriétés des modèles aux données disponibles sur le comportement des banques. Mimir (2011) ouvre la voie en ce domaine sur données américaines.

o de la persistance et l'ampleur du ralentissement du PIB dans les pays de l'OCDE. Les modèles prédisent une dynamique macroéconomique qui n'est pas comparée avec précision aux chiffres de la comptabilité nationale. Or, il s'agit d'un exercice qui a été conduit pour les crises du XXème siècle (voir le numéro spécial de la Review of Economic Dynamics, 2002, vol 5.)

- De plus, la dynamique macroéconomique des modèles est souvent présentée à la suite de chocs financiers. Cela appelle deux remarques.

o i) il est impossible de distinguer le rôle respectif de l'introduction d'un choc nouveau (choc financier) et de la mise en œuvre d'un nouveau mécanisme de transmission. Il faudrait pouvoir examiner les prédictions du modèle suite à un 
choc réel dont les effets sont bien connus (tel qu'un choc technologique) afin d'isoler l'effet d'amplification inhérent aux raffinements théoriques proposés.

o ii) les modèles affinant la modélisation du secteur financier proposent différents types de chocs financiers (choc sur la richesse nette des banques commerciales, choc sur la liquidité sur le marché interbancaire, choc sur la prime de risque des agents privés non financiers, choc sur l'incertitude,...). La recherche de fondements empiriques rigoureux à ces chocs pourrait faciliter l'identification des aléas les plus pertinents sur les marchés financiers dans la crise récente et donc l'identification des modélisations les plus intéressantes.

Il est donc difficile d'évaluer la pertinence quantitative de la réponse de l'économie à un choc dont l'ampleur n'a pas été calée sur des données.

- Enfin, l'apport quantitatif d'un modèle par rapport à ceux de la littérature n'est pas encore bien évalué. En particulier, on s'interroge sur l'impact quantitatif des nouveaux mécanismes décrits dans GK(2010) par rapport à ceux de $\operatorname{GKr}(2011)$ ou MM (2010). GKQ (2011), par l'introduction d'actions dans le passif de la banque, proposent un modèle qui tend à modérer l'effet d'amplification de GK(2010). Cette modération est-elle significative ? Cela reste à déterminer.

Une estimation structurelle de l'ensemble de ces modèles permettraient de répondre à ces questions et devrait faire l'objet de travaux futurs. De tels projets de recherche pourraient également contribuer à l'identification d'une politique monétaire robuste à la nature des chocs dans l'économie et au modèle retenu. Il s'agit également d'une étape indispensable dans la compréhension des fluctuations économiques dans la crise récente, alors que des économistes comme Cole (2011), Ohanian (2010) suggèrent que les frictions financières ne semblent pas avoir joué un rôle déterminant dans la crise, faisant écho aux travaux de Chari et al. (2010) qui, à partir de l'examen des données agrégés aux Etats-Unis, suggèrent que les entreprises s'auto-financent à hauteur de $80 \%$ et remettent en question l'idée de même d'un «credit crunch ».

Nous n'avons pas évoqué dans cet article les aspects internationaux qui mériteraient un article à part entière. Des études empiriques soulignent l'importance des transferts internationaux d'épargne dans la globalisation de la prise de risque (Brender et Pisani, 2010). Des développements MEGIS sur la contagion financière à l'échelle internationale ont donc vu le jour (voir le numéro spécial de la European Economic Review 2011 vol. 55, p. 307-442).

Malgré des avancées majeures, le tableau 2 indique qu'il reste à explorer 3 directions. La première viserait à développer la dimension non linéaire dans la dynamique macroéconomique et financière. Dans le modèle de GKQ (2010), on s'interroge sur la transition du régime à incertitude modérée à celui caractérisée par une forte incertitude. Les auteurs restent silencieux sur la possibilité du passage, peut-être brutal, d'un régime à l'autre. Les économistes intéressés par les pays émergents sont familiers des dynamiques «boombust » ou « sudden stop ». Cette dernière correspond à 2 régimes dans l'économie : l'embellie (phase au cours de laquelle les agents ne rencontrent aucune difficulté pour trouver des financements) suivie d'une catastrophe (les agents sont contraints sur leur financement). Cela correspond à une formalisation dans laquelle les agents subissent une contrainte de financement occasionnellement saturée (non saturée en phase d'embellie, brutalement saturée en phase de catastrophe), ce qui donne lieu à une complexité computationnelle bien supérieure à celle des articles évoqués dans cet article.

Les deux derniers champs de recherche concernent la titrisation et la prise en compte d'une mauvaise information des investisseurs sur les marchés financiers. Comme nous l'avons souligné dans la section 3.1. b), ces 2 éléments sont imbriqués. Les contributions de de 
Dubecq, Mojon et Ragot (2010) Faia (2011) et Chari, Shourideh, Zettlin-Jones (2010) explorent ce point. Toutefois, l'impact macroéconomique de ces deux éléments reste à analyser.

\section{Références bibliographiques}

Adrian, T., Ashcraft, A., Boesky, H., \& Pozsar, Z. (2010). Shadow Banking. Federal Reserve Bank of New York Staff Reports 458, Federal Reserve Bank of New York.

Adrian, T. \& Shin, H. (2010). Financial intermediaries and monetary economics. Dans B. M. Friedman \& M. Woodford (Eds.), Handbook of Monetary Economics, volume 3 (pp.601650).: Elsevier.

Akerlof, G. A. \& Shiller, R. (2009). Animal Spirits. Princeton University Press.

Allen, F. \& Gale, D. (1994). Limited market participation and volatility of asset prices. American Economic Review, 84(4), 933-955.

Allen, F. \& Gale, D. (2000). Bubbles and crises. Economic Journal, 110, 236-255.

Allen, F. \& Gale, D. (2007). Understanding Financial Crises. Oxford: Oxford University Press.

Angeloni, I. \& Faia, E. (2010). Capital Regulation and Monetary Policy with Fragile Banks. Mimeo, Goethe University Frankfurt.

Banque de France (2009). La Crise Financière. Documents et Débats n², Banque de France.

Bank of England (2009). The Role of Macroprudential Policy. Bank of england discussion paper, Bank of England.

BCE (2009). Bulletin Mensuel. Avril.

Bernanke, B. (2004). The Great Moderation. Remarks by governor ben bernanke at the meetings of the eastern economic association, washington, dc, Fed.

Bernanke, B. (2009). The Crisis and the Policy Response. Speech at the fed, Fed.

Bernanke, B. \& Blinder, A. (1988). Credit, money, and aggregate demand. American Economic Review, 78(2), 435-439.

Bernanke, B. \& Gertler, M. (1995). Inside the black box: The credit channel of monetary policy transmission. The Journal of Economic Perspectives, 9(4), 27-48.

Bernanke, B., Gertler, M., \& Gilchrist, S. (1999). The financial accelerator in a quantitative business cycle framework. Dans J. B. Taylor \& M. Woodford (Eds.), Handbook of Macroeconomics, volume 1 (pp.1341-1393).: Elservier.

Blanchard, O. \& Gali, J. (2007). Real wage rigidities and the new keynesian model. Journal of Money, Credit, and Banking, 39(1), 35-65. 
Blot, C. \& Timbeau, X. (2009). Du chaos financier au k.o. économique. Revue de l'OFCE, 2009/3(110), 149-178.

Borio, C. \& Zhu, H. (2008). Capital Regulation, Risk-Taking and Monetary Policy: A Missing Link in the Transmission Mechanism? BIS Working Papers 268, BIS.

Brender, A. \& Pisani, F. (2010). La crise de la finance globalisée. Economie et Statistique, 438-440, 85-104.

Browning, M. \& Tobacman, J. (2007). Discounting and Optimism Equivalences. Mimeo, Oxford.

Brunnermeier, M. (2009). Deciphering the liquidity and credit crunch 2007-08. Journal of Economic Perspectives, 23(1), 77-100.

Buiter, W. (2009). The Unfortunate Uselessness of Most 'state of the Art' Academic Monetary Economics. Mimeo.

Caballero, J. (2010). Macroeconomics after the crisis: Time to deal with the pretense-ofknowledge syndrome. Journal of Economic Perspectives, 24(4), 85-102.

CAE (2008). La crise des subprimes. Dans P. Artus, J. Betbèze, C. DeBoissieu, \& G. CapelleBlancard (Eds.), Rapport Conseil d'Analyse Economique.

CAE (2011). Banques centrales et stabilité financière. Dans J. Betbeze, C. Bordes, J. Couppey-Soubeyran, \& D. Plihon (Eds.), Rapport Conseil d'Analyse Economique.

Cagetti, M. (2003). Accumulation over the life cycle and precautionnary saving. Journal of Business and Economic Statistics, 21, 339-353.

Calza, L., Monacelli, T., \& Stracca, L. (2009). Housing Finance and Monetary Policy. ECB Working Paper 1069, ECB.

Capelle-Blancard, G. \& Couppey-Soubeyran, J. (2003). Le financement des agents non financiers en europe : Le rôle des intermédiaires financiers demeure prépondérant. Economie et Statistiques, (366).

Carlstrom, C. \& Fuerst, T. (1997). Agency costs, net worth, and business fluctuations: A computable general equilibrium analysis. The American Economic Review, 87(5), 893- 910.

Carlstrom, C. \& Fuerst, T. (1998). Agency cost and business cycles. Economic Theory, 12, 583-597.

Carroll, C. (2000). Requiem for the representative consumer? aggregate implications of microeconomic consumption behavior. American Economic Review, 90(2), 110-115.

Chari, V., Christiano, L., \& Kehoe, P. (2008). Facts and Myths About the Financial Crisis of 2008. Federal Reserve Bank of Minneapolis Working Paper 666, Federal Reserve Bank of Minneapolis. 
Chari, V., Shourideh, A., \& Zetlin-Jones, A. (2010). Damage Control? Analyzing Policies to Repair Credit Markets. Economic Policy Paper 10-5, Federal Reserve Bank of Minneapolis.

Chatelain, J., Generale, A., Hernando, I., Vermeulen, P., \& Von Kalckreuth, U. (2003a). Firm investment and monetary policy transmission in france. Dans I. Angeloni, I. Kashyap, \& B. Mojon (Eds.), Monetary Policy Transmission in the Euro Area: Cambridge University Press.

Chatelain, J., Generale, A., Hernando, I., Vermeulen, P., \& Von Kalckreuth, U. (2003b). Firm investment and monetary policy transmission in the euro area. Dans I. Angeloni, I. Kashyap, \& B. Mojon (Eds.), Monetary Policy Transmission in the Euro Area: Cambridge University Press.

Christensen, I.and Dib, A. (2008). The financial accelerator in an estimated new keynesian model. Review of Economic Dynamics, 11, 155-178.

Christiano, L., Ilut, C., Motto, R., \& Rostagno, M. (2010). Onetary Policy and Stock Market Booms. NBER Working Paper 16402, NBER.

Christiano, L., Motto, R., \& Rostagno, M. (2010). Financial Factors in Economic Fluctuations. ECB working paper 1192, ECB.

Coeurdacier, N., Rey, H., \& Winant, P. (2011). The risky steady state. American Economic Review, 101(3), 398-401.

Cole, H. (2011). Discussion of gertler and karadi : A model of unconventional monetary policy. Journal of Monetary Economics, 58, 35-38.

Curdia, V. \& Woodford, M. (2010). Conventional and unconventional monetary policy. Federal Reserve Bank of St. Louis Review.

DeGraeve, F. (2008). The external finance premium and the macroeconomy: US post-WWII evidence. Journal of Economic Dynamics and Control, 32(11), 3415-3440.

DeGrauwe, P. (2009). Animal Spirits and Monetary Policy. CESifo Working Paper 2418, CESifo.

Diamond, D. \& Rajan, R. (2000). A theory of bank capital. Journal of Finance, 55(6), 24312465.

Dubecq, S., Mojon, B., \& Ragot, X. (2010). Fuzzy Capital Requirements, Risk-Shifting and the Risk Taking Channel of Monetary Policy. Mimeo, Banque de France.

Elsby, M., Hobijn, B., \& Sahin, A. (2010). The labor market in the great recession. Brookings Papers on Economic Activity, 41(1), 1-69.

Faia, E. (2011). Credit Risk Transfers and the Macroeconomy. Kiel Working Paper 1677, Kiel Institute for the World Economy. 
Faia, E. \& Monacelli, T. (2005). Optimal Monetary Policy Rules, Asset Prices and Credit Frictions. CEPR Discussion Paper 4880, CEPR.

Galati, G. \& Moessner, R. (2011). Macroprudential Policy a Literature Review. BIS Working Paper 337, BIS.

Gelain, P. (2010). The external finance premium in the euro area: A dynamic stochastic general equilibrium analysis. North American Journal of Economics and Finance, 21, 49-71.

Gertler, M. (2010). Banking crises and real activity: Identifying the linkages. LinkagesInternational Journal of Central Banking, 6(34), 125-135.

Gertler, M. \& Karadi, P. (2011). A model of unconventional monetary policy. Journal of Monetary Economics, 58, 17-34.

Gertler, M. \& Kiyotaki, N. (2010). Financial intermediation and credit policy in business cycle analysis. Dans B. Friedman \& M. Woodford (Eds.), Handbook of Monetary Economics, volume 3 (pp.547-599).: Elsevier.

Gertler, M., Kiyotaki, N., \& Queralto, A. (2010). Financial Crises, Bank Risk Exposure and Government Financial Policy. Mimeo, New York University.

Ghironi, F., Iscan, T., \& Rebucci, A. (2008). Net foreign asset positions and consumption dynamics in the international economy. Journal of International Money and Finance, 27, 1337-1359.

Gilchrist, S. \& Zakrajsek, E. (2011). Credit spreads and business cycle fluctuations. Amerian Economic Review.

Goodfriend, M. (2009). Central Banking in the Credit Turmoil: An Assessment of Federal Reserve Practice. Mimeo, Carnegie Mellon.

Goodhart, C. (2011). La supervision macro-prudentielle. Dans J. Betbèze, C. Bordes, J. Couppey-Soubeyran, \& D. Plihon (Eds.), Banques Centrales et Stabilité Financière (pp.295316). Paris: Rapport CAE.

Hanson, S., Kashyap, A., \& Stein, J. (2011). A macroprudential approach to financial regulation. Journal of Economic Perspectives, 25(1), 3-28.

Iacoviello, M. (2005). House prices, borrowing constraints and monetary policy in the business cycle. American Economic Review, 95(3), 739-764.

Iacoviello, M. (2010). Housing In DSGE models: Findings and new directions. Dans O. DeBandt, T. Knetsch, \& J. Penalosa (Eds.), Housing Markets In Europe : A Macroeconomic Perspective (pp.3-18). Berlin: Springer-Verlag.

Iacoviello, M. \& Neri, S. (2010). Housing market spillovers: Evidence from an estimated DSGE model. American Economic Journal: Macroeconomics, 2(2), 125-164. 
IMF (2008). The housing sector and the business cycle. Dans IMF (Ed.), World Economic Outlook Washington DC: IMF.

IMF (2009). Financial Stability Report. Washington DC: IMF.

Kannan, P. (2010). Credit Conditions and Recoveries from Recessions Associated with Financial Crises. IMF Working Paper WP/10/83, IMF.

Kiyotaki, N. \& Moore, J. (1997). Credit cycles. Journal of Political Economy, 105, 211-248.

Krusell, P. \& Smith, A. (1998). Income and wealth heterogeneity In the macroeconomy. Journal of Political Economy, 106(5), 867-896.

Landau, J. (2009). Bubbles and Macro Prudential Supervision. Speech by j.p. landau, deputy governor of the Bank of France, at the at the joint conference on "the future of financial regulation", Banque de France.

Lawrance, E. (1991). Poverty and the rate of time preference: Evidence from panel data. Journal of Political Economy, 99, 54-77.

Levieuge, G. (2005). Les banques comme vecteurs et amplificateurs des chocs financiers : Le canal du capital bancaire. Economie internationale, 2005/4(104), 65-95.

Levieuge, G. (2009). The bank capital channel and counter-cyclical prudential regulation in a DSGE model. Recherches économiques de Louvain, 2009/4(75), 425-460.

Meh, C. \& Moran, K. (2010). The role of bank capital in the propagation of shocks. Journal of Economic Dynamics and Control, 34(3), 555-576.

Mésonnier, J. (2005). Capitalisation Bancaire et Transmission de la Politique Monétaire : Une Revue. Mimeo.

Mian, A. \& Su, A. (2009). Household Leverage and the Recession of 2007 to 2009. Mimeo, University of Chicago.

Miller, M. \& Stiglitz, J. (2010). Leverage and Asset Bubbles: Averting Armageddon with Chapter 11? NBER Working Paper 15817, NBER.

Mimir, Y. (2011). Financial Intermediaries, Leverage Ratios and Business Cycles. Mimeo, University of Maryland.

Ohanian, L. (2010). The economic crisis from a neoclassical perspective. Journal of Economic Perspectives, 24(4), 1-24.

QueijoVonHeideken, V. (2008). How Important are Financial Frictions In the US and the Euro Area. Mimeo.

Rajan, R. (2005). Has Financial Development Made the World Riskier? NBER Working Paper 11728, NBER. 
Solomon, B. (2010). Firm Leverage, Household Leverage and the Business Cycle. Mimeo, UQAM.

Stock, J. \& Watson, M. (2002). Has the business cycle changed and why? Dans M. Gertler \& K. Rogoff (Eds.), NBER Macroeconomics Annual, volume 17 (pp.159-230).: NBER.

Taylor, J. \& Wieland, W. (2009). Surprising Comparative Properties of Monetary Models: Results from a New Data Base. NBER Working Paper 14849, NBER.

VanDenHeuvel, P. (2002). Does bank capital matter for monetary transmission. Federal Reserve Bank of New York Economic Policy Review.

Wikens, M. (2009). What's Wrong with Modern Macroeconomics? Why its Critics Have Missed the Point. Cesifo working paper, CESifo.

\section{ANNEXES}

\section{Annexe A : BGG, Remarques sur la résolution du modèle}

BGG intègrent ce contrat de dette dans un modèle d'équilibre général permettant de quantifier l'interaction entre les facteurs financiers et les variables macroéconomiques. Une telle tâche requiert de résoudre plusieurs difficultés. La première concerne l'agrégation des entrepreneurs. La seconde a trait à l'intégration du contrat de dette dans un modèle macroéconomique.

Tout d'abord, les chocs macroéconomiques peuvent affecter la solvabilité des emprunteurs. Le contrat de dette doit donc dépendre, non seulement du choc spécifique à chaque entrepreneur, mais également du choc agrégé, affectant l'ensemble de l'économie. De plus, les entrepreneurs sont caractérisés par une distribution de richesses nettes. En l'absence d'hypothèses simplificatrices, cela contraindrait l'économiste à calculer à chaque date la distribution des richesses nettes, tâche impliquant des temps de calcul considérables. L'agrégation de la richesse nette à l'échelle macroéconomique est alors simplifiée par l'hypothèse de neutralité au risque des entrepreneurs. En effet, la neutralité au risque rend les décisions de chaque entrepreneur linéaire. L'agrégation des comportements des entrepreneurs nécessite simplement la somme des décisions individuelles. Le comportement agrégé des entrepreneurs, résumés par des expressions linéaires, a donc la même forme que la décision individuelle. Cette hypothèse est courante et vise à simplifier le calcul de la richesse agrégée des entrepreneurs. L'abandon de la neutralité au risque obligerait l'économiste à suivre la dynamique de la distribution de richesse dans un environnement avec des entrepreneurs hétérogènes. BGG présentent une version de leur modèle avec deux types d'entrepreneurs, les premiers subissant de plus fortes contraintes de financement (une prime de financement externe plus élevée) que les seconds. Des travaux récents proposent des modèles avec un continuum de firmes hétérogènes et montrent que l'hétérogénéité introduit un canal supplémentaire de propagation des chocs. Nous présentons dans cet article l'accélérateur financier dans un cadre simple afin d'illustrer ce mécanisme économique de manière transparente. 
BGG supposent également qu'une proportion exogène des entrepreneurs fait faillite à chaque période. ${ }^{29}$ Cette hypothèse évite l'occurrence dans le modèle d'une richesse nette si abondante que l'investissement ne nécessiterait jamais un financement par endettement. Cela vient rappeler que le taux d'endettement règle l'ampleur des effets de l'accélérateur financier. Une économie endettée est soumise aux effets d'amplification inhérents au modèle de BGG. Cette hypothèse est courante et reprise dans les articles ultérieurs lorsque l'emprunteur est une banque (Gertler et Karadi, 2009 ; Angeloni et Faia, 2010).

Le stock de richesse nette agrégée s'écrit comme la différence entre le stock de capital, évalué à son prix de marché et augmenté de son rendement, et le remboursement de la dette (auquel s'applique la prime de financement externe). A ces termes s'ajoute le salaire de l'entrepreneur dans l'activité productive. Cette hypothèse est nécessaire car les entrepreneurs voient leur capital confisqué par la banque en cas de défaut de paiement. Le salaire leur permet d'accumuler à nouveau une richesse nette. Grâce à cette dernière, des emprunts bancaires sont envisageables à nouveau pour redémarrer un projet d'investissement

Par cette relation agrégée, le stock de richesse nette est affecté par l'inflation, le prix des actifs ainsi que les conditions d'accès au crédit résumées par la prime de financement externe. L'ampleur de ces effets est réglée par le degré d'endettement de l'économie.

\section{Annexe B : CW, Remarques sur la résolution du modèle}

Il y a deux types d'agents ( $b$ et $s$ ) : ceux qui dérivent à chaque période l'utilité marginale à la consommation Ucb, et ceux qui en dérivent Ucs, avec Ucb > Ucs. Cette hypothèse ${ }^{30}$ implique que les agents du type $b$ valorisent les dépenses courantes davantage que les dépenses futures; ils sont donc plus impatients que les agents de type $s$ et disposés à payer davantage pour emprunter. De plus, l'étalonnage du modèle est tel que, à l'équilibre, les agents de type $b$ sont endettés et ceux de type s sont créditeurs. L'hypothèse centrale, source de la friction financière, concerne la possibilité d'épargne et d'emprunt des ménages. Ces derniers doivent obligatoirement passer par la banque pour leurs opérations financières d'épargne ou d'emprunt. CW supposent également l'accès aux titres de la dette de l'Etat mais, en l'absence d'autres frictions financières, le rendement de ces titres publics est égal à celui des dépôts bancaires. La structure des taux d'intérêts dépend donc, in fine, du secteur d'intermédiation.

Cette structure hétérogène des agents pourrait engendrer une grande complexité computationnelle à l'équilibre général. Afin de la contourner, des hypothèses supplémentaires sont faites de façon à ce que l'histoire de chaque individu ${ }^{31}$ n'affecte pas la richesse, cette dernière dépendant uniquement du type de l'individu à la période courante. La dynamique agrégée peut donc être résumée par l'évolution des utilités marginales des 2 types d'agent.

Les hypothèses simplifiant l'agrégation des comportements sont les suivantes. Les habitants de cette économie sont soumis tout d'abord à un risque idiosyncrasique qui les fait changer de type selon une probabilité fixe. En outre, à chaque période, seulement ceux qui changent de

\footnotetext{
${ }^{29}$ Le nombre de faillites dans le modèle de BGG comporte donc deux composantes : une composante exogène liée à l'hypothèse évoquée ci-dessus et une composante endogène consécutive à des chocs agrégés négatifs.

${ }^{30}$ De plus, les agents ont une désutilité marginale du travail différente de façon à avoir une même quantité de travail à l'état stationnaire pour les 2 types de ménages. Aussi, CW supposent-ils que les agents type $b$ ont un degré de substitution intertemporelle supérieure à celui des agents du type $s$.

${ }^{31}$ L'histoire de l'individu signifie la séquence complète des chocs qui l'ont affecté depuis l'origine des temps. Si cette histoire compte dans les choix individuels présents et futurs, il faudrait, dans la résolution du modèle, garder en mémoire l'histoire individuelle ; ce qui introduit une lourdeur computationnelle significative.
} 
type ont la possibilité de recevoir le paiement associé à un contrat d'assurance contre le risque idiosyncrasique de changement de type ainsi que contre le risque agrégé. La possibilité de recevoir les indemnités associées à cette assurance est toutefois intermittente. L'accès à l'assurance est limité aux périodes où les agents changent de type. Entre ces périodes d'accès à l'assurance, les agents peuvent épargner ou emprunter uniquement auprès des intermédiaires financiers. Ces hypothèses permettent d'avoir une structure de ménages dont l'hétérogénéité est limitée à 2 types. La distribution de richesse est résumée uniquement par l'évolution de 2 utilités marginales grâce à une structure de marchés financiers complets. Toutefois, ces marchés complets étant intermittents, la présence des banques s'avère nécessaire.

\section{Annexe C. GK, la contrainte de non faillite de la banque dans un modèle à 2 périodes}

Le bilan de la banque se compose au passif

- des dépôts des ménages $d$ rémunés au facteur d'intérêt $R$

- de la richesse nette des banques $N$

- de la dette interbancaire $b^{i}$ avec $R^{i}$ le facteur d'intérêt associé

et à l'actif, la banque octroie des crédits aux (achète des actions émise par) les entrepreneurs pour un montant $L$ dont le rendement est $R^{k}$.

L'équilibre actif-passif implique $L=\left(N+d+b^{i}\right)$

- Le profit de la banque en cas de non faillite est $\Pi_{N F}=\left(N+d+b^{i}\right) R^{k}-d R-b^{i} R^{i}$

- Le profit de la banque en cas de faillite est $\Pi_{F}=\theta R^{k}\left[N+d+b^{i}(1-\omega)\right]$

La contrainte de participation de la banque peut s'écrire :

$$
\Pi_{N F} \geq \Pi_{F}
$$

soit

ou encore

$$
\left(N+d+b^{i}\right) R^{k}-d R-b^{i} R^{i} \geq \theta R^{k}\left[N+d+b^{i}(1-\omega)\right]
$$

$$
(1-\theta) R^{k} N \geq\left[\theta R^{k}-\left(R^{k}-R\right)\right] d+\left[\theta R^{k}(1-\omega)-\left(R^{k}-R^{i}\right)\right] b^{i}
$$

La banque ne fait pas faillite si le terme de droite est peu élevé, donc si les spreads $\left(R^{k}-R\right)$ et $\left(R^{k}-R^{i}\right)$ sont élevés et/ou $d$ et $b^{i}$ faibles. Ces spreads doivent être d'autant plus élevés que les frictions financières sont fortes ( $\theta$ grand ou $\omega$ faible).

\section{Annexe D : GKQ, Remarques sur l'équilibre de long terme augmenté du risque}

Le modèle de GKQ place le risque au centre de son analyse. La résolution du modèle requiert d'expliciter l'impact de l'incertitude de l'économie (mesurée par la volatilité) sur les décisions des agents et l'équilibre de long terme de l'économie. Ce qui explique que les choix des agents diffèrent selon le degré d'incertitude dans l'économie (incertitude modérée, étalonnée pour reproduire la volatilité observée aux Etats-Unis pendant la Grande Modération, versus incertitude marquée, étalonnée sur la période 1960-1980).

GKQ calculent l'équilibre de long terme augmenté du risque (« risk adjusted steady state »). Dans les MEGIS standard, l'équilibre de long terme caractérise l'évolution de l'économie en l'absence de chocs (environnement déterministe dans lequel l'état stationnaire ne dépend que des valeurs macroéconomiques moyennes). Les variables du modèle (purgées de la tendance technologique déterministe) sont constantes. En revanche, dans l'équilibre de long terme augmenté du risque, les variables du modèle dépendent également des moments de second 
ordre (variances et co-variances). Cela traduit la prise en compte de l'environnement stochastique à long terme. L'équilibre de long terme dans l'économie avec incertitude modérée diffère donc de celui qui caractérise l'économie avec incertitude marquée.

Puisque l'équilibre de long terme dépend des volatilités, ces dernières affectent les décisions des agents (les élasticités déterminées après log - linéarisation des conditions d'optimalité) et donc l'évolution et la volatilité de l'ensemble des variables par des effets d'équilibre général, volatilités qui elles-mêmes affectent l'équilibre de long terme. La résolution du modèle requiert donc la recherche d'un point fixe par une approche itérative :

1. log - linéarisation des équations du modèle autour de l'équilibre de long terme déterministe

2. simulation du modèle soumis à des chocs (dont la volatilité est étalonnée sur la période d'incertitude forte ou modérée) et calcul des moments du $2^{\text {nd }}$ ordre de toutes les variables

3. calcul de l'équilibre de long terme augmenté du risque à l'aide des moments obtenus en étape 2 .

4. log - linéarisation des équations du modèle autour de l'équilibre de long terme augmenté du risque. ${ }^{32}$

5. revenir à étape 2. Répéter jusqu'à coïncidence entre moments du $2^{\text {nd }}$ ordre de toutes les variables entre 2 itérations successives (cela montrera la cohérence entre les moments du $2^{\text {nd }}$ ordre engendrés par le modèle après simulation et ceux utilisés pour calculer l'équilibre de long terme).

\footnotetext{
${ }^{32}$ Le choix du point autour duquel le modèle est linéarisé est central car il affecte la dynamique des variables. Cela revient à déterminer les conditions initiales de la dynamique macroéconomique. Dans les MEGIS standard, l'équilibre de long terme définit les conditions initiales. Voir la discussion dans Coeurdacier, Rey et Winant (2011).
} 


\begin{tabular}{|c|c|c|c|c|c|c|c|c|}
\hline \multicolumn{2}{|c|}{ point central de l'article } & accélérateur financier & $\begin{array}{l}\text { accélérateur financier dans un } \\
\text { canal large du crédit }\end{array}$ & spread de taux & $\begin{array}{l}\text { canal du capital } \\
\text { bancaire }\end{array}$ & \begin{tabular}{|l} 
Le canal \\
du crédit \\
inter-bancaire \\
\end{tabular} & $\begin{array}{l}\text { canal de la prise de } \\
\text { risque }\end{array}$ & $\begin{array}{l}\text { canal de la prise } \\
\text { de risque }\end{array}$ \\
\hline \multicolumn{2}{|l|}{ article de référence } & $\begin{array}{l}\text { Kiyotaki et Moore (1997) } \\
\text { actualisés par lacoviello } \\
\text { (2005) }\end{array}$ & $\begin{array}{l}\text { Bernanke, Gertler et Gilchrist } \\
\text { (1999) }\end{array}$ & $\begin{array}{l}\text { Curdia } \\
\text { et } \\
\text { Woodford } \\
(2010)\end{array}$ & $\begin{array}{l}\text { Meh et Moran (2010), } \\
\text { Levieuge (2009), } \\
\text { Gertler et Karadi } \\
(2011)\end{array}$ & Gertler et Kiyotaki (2010) & $\begin{array}{l}\text { Gertler, Kiyotaki et } \\
\text { Queralto (2010) }\end{array}$ & $\begin{array}{l}\text { Angeloni et Faia } \\
\text { (2010), Angeloni, } \\
\text { Faia et Winkler } \\
\text { (2010) }\end{array}$ \\
\hline \multicolumn{2}{|c|}{ mécanisme économique } & $\begin{array}{l}\text { accélérateur financier: } \\
\text { impact du prix des actifs } \\
\text { sur le collateral donc sur } \\
\text { la contrainte } \\
\text { d'endettement des } \\
\text { ménages et des } \\
\text { entreprises; taux } \\
\text { d'endettement exogène }\end{array}$ & $\begin{array}{l}\text { accélérateur financier sur le bilan } \\
\text { des entreprises et des ménages } \\
\text { + contrat de dette donc taux } \\
\text { d'endettement endogène }\end{array}$ & $\begin{array}{l}\text { choc financier sur les } \\
\text { coûts de la banque donc } \\
\text { baisse des crédits et } \\
\text { hausse du spread }\end{array}$ & $\begin{array}{l}\text { accélérateur financier } \\
\text { sur le capital bancaire }\end{array}$ & \begin{tabular}{|l|} 
hétérogénéité des besoins \\
de financement des \\
banques, certaines sont \\
mises en difficulté car elles \\
n'ont pas accès aux \\
financements
\end{tabular} & \begin{tabular}{|l|} 
décision de la banque \\
sur la composition de \\
son passif: le recours \\
massif à la dette de \\
court terme en période \\
d'incertitude modérée \\
rend le secteur bancaire \\
sensible aux chocs \\
agrégés \\
\end{tabular} & $\begin{array}{l}\text { La banque est } \\
\text { sujette à une } \\
\text { possibilité de } \\
\text { panique bancaire, } \\
\text { ce qui l'incite à } \\
\text { disposer de fonds } \\
\text { propres }\end{array}$ \\
\hline \multicolumn{2}{|l|}{ Traits marquants } & $\begin{array}{l}\text { Contrainte d'endettement } \\
\text { exogène, justifiée par un } \\
\text { limited enforcement ; } \\
\text { Accent sur la richesse } \\
\text { immobilière }\end{array}$ & $\begin{array}{l}\text { Prime de financement externe } \\
\text { décroissante avec la richesse } \\
\text { nette de l'emprunteur. Le contrat } \\
\text { de dette est fidèle aux faits }\end{array}$ & $\begin{array}{l}\text { coûts de la banque } \\
\text { proportionnels au volume } \\
\text { de crédits et des } \\
\text { réserves; spread de taux } \\
\text { est une fonction } \\
\text { croissante du volume de } \\
\text { crédits et décroissante } \\
\text { des réserves }\end{array}$ & $\begin{array}{l}\text { la banque doit } \\
\text { disposer de fonds } \\
\text { propres de façon à } \\
\text { convaincre les } \\
\text { ménages de lui } \\
\text { confier ses avoirs }\end{array}$ & $\begin{array}{l}\text { la banque doit disposer de } \\
\text { fonds propres de façon à } \\
\text { convaincre les ménages de } \\
\text { lui confier ses avoirs et les } \\
\text { autres banques de lui } \\
\text { fournir des financements }\end{array}$ & $\begin{array}{l}\text { passif de la banque } \\
\text { composé d'actions, de } \\
\text { fonds propres et de } \\
\text { dépôts des ménages }\end{array}$ & $\begin{array}{l}\text { possibilité de } \\
\text { panique bancaire : } \\
\text { retrait des dépôts } \\
\text { des ménages }\end{array}$ \\
\hline \multicolumn{2}{|c|}{$\begin{array}{l}\text { Défaut de paiement des agents non } \\
\text { financiers (endogène) }\end{array}$} & non & oui & non & oui & non & non & non \\
\hline \multirow{2}{*}{$\begin{array}{l}\text { composition du } \\
\text { bilan de la banque } \\
\text { commerciale }\end{array}$} & actif & prêts aux entrepreneurs & prêts aux entrepreneurs & $\begin{array}{l}\text { prêts aux entrepreneurs } \\
\text { et réserves auprès de la } \\
\text { banque centrale }\end{array}$ & \begin{tabular}{|l} 
prêts aux \\
entrepreneurs ou \\
actions émises par \\
les entrepreneurs \\
(GKr) \\
\end{tabular} & $\begin{array}{l}\text { actions émises par les } \\
\text { entrepreneurs }\end{array}$ & $\begin{array}{l}\text { actions émises par les } \\
\text { entrepreneurs }\end{array}$ & $\begin{array}{l}\text { prêts aux } \\
\text { entrepreneurs }\end{array}$ \\
\hline & passif & dépôt des ménages & dépôt des ménages & dépôt des ménages & $\begin{array}{l}\text { dépôt des ménages } \\
\text { (aléa moral), fonds } \\
\text { propres }\end{array}$ & $\begin{array}{l}\text { dépôt des ménages (aléa } \\
\text { moral), fonds propres et } \\
\text { dette interbancaire (aléa } \\
\text { moral) }\end{array}$ & $\begin{array}{l}\text { dépôt des ménages } \\
\text { (aléa moral), fonds } \\
\text { propres et actions } \\
\text { émises par la banque } \\
\end{array}$ & $\begin{array}{l}\text { dépôt des } \\
\text { ménages (bank } \\
\text { run), fonds propres }\end{array}$ \\
\hline \multicolumn{2}{|c|}{$\begin{array}{l}\text { Est-ce que la banque optimise son bilan? } \\
\text { Prend-t-elle des décisions sur son bilan? }\end{array}$} & non & non & oui & oui & oui & oui & oui \\
\hline \multirow{3}{*}{$\begin{array}{l}\text { Défaut de paiement } \\
\text { des banques } \\
\text { (endogène) }\end{array}$} & $\begin{array}{l}\text { possibilité de défaut de } \\
\text { paiement }\end{array}$ & non & non & non & oui & oui & oui & oui \\
\hline & $\begin{array}{l}\text { défaut de paiement à } \\
\text { l'équilibre }\end{array}$ & & & non & non & non & non & oui \\
\hline & règle de Taylor & oui & oui & oui & oui & oui & Inon & oui \\
\hline
\end{tabular}




\begin{tabular}{|c|c|c|c|c|c|c|c|c|}
\hline \multicolumn{2}{|c|}{ point central de l'article } & accélérateur financier & $\begin{array}{l}\text { accélérateur financier dans un } \\
\text { canal large du crédit }\end{array}$ & spread de taux & $\begin{array}{l}\text { canal du capital } \\
\text { bancaire }\end{array}$ & \begin{tabular}{|l} 
Le canal \\
du crédit \\
inter-bancaire \\
\end{tabular} & $\begin{array}{l}\text { canal de la prise de } \\
\text { risque }\end{array}$ & $\begin{array}{l}\text { canal de la prise } \\
\text { de risque }\end{array}$ \\
\hline \multicolumn{2}{|c|}{ article de référence } & $\begin{array}{l}\text { Kiyotaki et Moore (1997) } \\
\text { actualisés par lacoviello } \\
\text { (2005) }\end{array}$ & $\begin{array}{l}\text { Bernanke, Gertler et Gilchrist } \\
\text { (1999) }\end{array}$ & $\begin{array}{l}\text { Curdia } \\
\text { et } \\
\text { Woodford } \\
(2010)\end{array}$ & $\begin{array}{l}\text { Meh et Moran (2010), } \\
\text { Levieuge (2009), } \\
\text { Gertler et Karadi } \\
(2011)\end{array}$ & Gertler et Kiyotaki (2010) & $\begin{array}{l}\text { Gertler, Kiyotaki et } \\
\text { Queralto (2010) }\end{array}$ & $\begin{array}{l}\text { Angeloni et Faia } \\
\text { (2010), Angeloni, } \\
\text { Faia et Winkler } \\
(2010)\end{array}$ \\
\hline Banque centrale & $\begin{array}{l}\text { politique non } \\
\text { conventionnelle } \\
\text { (Quantitative Easing } \\
\text { QE; Credit Easing CE) }\end{array}$ & non & non & oui (QE et CE) & $\begin{array}{l}\text { non sauf } \mathrm{GKr}(2011) \\
\mathrm{CE}\end{array}$ & oui (CE) & oui, CE & non \\
\hline \multicolumn{2}{|c|}{$\begin{array}{l}\text { Expressions sur lesquelles on ne doit pas } \\
\text { buter }\end{array}$} & $\begin{array}{l}\text { limite exogène sur le } \\
\text { crédit disponible; } \\
\text { redistribution de richesse } \\
\text { entre prêteur et } \\
\text { emprunteur via l'inflation }\end{array}$ & $\begin{array}{l}\text { Contrat de dette qui implique que } \\
\text { la prime de financement externe } \\
\text { (le spread de taux) est une } \\
\text { fonction croissante du ratio } \\
\text { d'endettement }\end{array}$ & $\begin{array}{l}\text { coûts de la banque } \\
\text { proportionnels au volume } \\
\text { de crédits et au montant } \\
\text { des réserves; spread de } \\
\text { taux est une fonction } \\
\text { croissante du volume de } \\
\text { crédits et décroissante } \\
\text { des réserves }\end{array}$ & $\begin{array}{l}\text { contrainte de non } \\
\text { faillite de la banque } \\
\text { sachant que ses } \\
\text { créanciers sont les } \\
\text { ménages }\end{array}$ & $\begin{array}{l}\text { contrainte de non faillite de } \\
\text { la banque sachant que ses } \\
\text { créanciers sont les } \\
\text { ménages et les autres } \\
\text { banques }\end{array}$ & $\begin{array}{l}\text { contrainte de non faillite } \\
\text { de la banque avec } \\
\text { endogénéité de la part } \\
\text { que la banque peut } \\
\text { s'approprier en cas de } \\
\text { faillite; équilibre de long } \\
\text { terme ajusté pour le } \\
\text { degré d'incertitude dans } \\
\text { l'économie }\end{array}$ & $\begin{array}{l}\text { partage de la } \\
\text { valeur liquidative } \\
\text { de la banque en } \\
\text { cas de faillite }\end{array}$ \\
\hline \multicolumn{2}{|c|}{ Modalité « ad hoc » } & $\begin{array}{l}\text { Hétérogénéité } \\
\text { des taux d'escompte } \\
\text { des agents }\end{array}$ & Entrepreneurs neutres au risque & $\begin{array}{l}\text { Hétérogénéité des } \\
\text { préférences; le spread } \\
\text { de taux est une fonction } \\
\text { croissante du volume } \\
\text { des crédits distribués et } \\
\text { décroissante des } \\
\text { réserves }\end{array}$ & \begin{tabular}{|l|} 
part que la banque \\
peut s'approprier en \\
cas de faillite est \\
exogène, donc, ne \\
varie pas au cours du \\
cycle économique
\end{tabular} & \begin{tabular}{|l|} 
Les difficultés de \\
financement interbancaire \\
sont liées à une \\
segmentation des marchés \\
financiers (modélisée par \\
les îles)
\end{tabular} & $\begin{array}{l}\text { part que la banque peut } \\
\text { s'approprier en cas de } \\
\text { faillite est croissante } \\
\text { dans le ratio actions / } \\
\text { total actif }\end{array}$ & $\begin{array}{l}\text { partage de la } \\
\text { valeur liquidative } \\
\text { de la banque en } \\
\text { cas de faillite } \\
\text { dépend de } \\
\text { manière exogène } \\
\text { du pouvoir de } \\
\text { négociation des } \\
\text { créanciers de la } \\
\text { banque (ménages } \\
\text { et actionnaires) }\end{array}$ \\
\hline
\end{tabular}

Page 2 de 2 